\title{
TU/e emonownen

\section{A computer checked algebraic verification of a distributed summation algorithm}

\section{Citation for published version (APA):}

Groote, J. F., Monin, F. G., \& Springintveld, J. (1997). A computer checked algebraic verification of a distributed summation algorithm. (Computing science reports; Vol. 9714). Technische Universiteit Eindhoven.

\section{Document status and date:}

Published: 01/01/1997

\section{Document Version:}

Publisher's PDF, also known as Version of Record (includes final page, issue and volume numbers)

\section{Please check the document version of this publication:}

- A submitted manuscript is the version of the article upon submission and before peer-review. There can be important differences between the submitted version and the official published version of record. People interested in the research are advised to contact the author for the final version of the publication, or visit the $\mathrm{DOI}$ to the publisher's website.

- The final author version and the galley proof are versions of the publication after peer review.

- The final published version features the final layout of the paper including the volume, issue and page numbers.

Link to publication

\section{General rights}

Copyright and moral rights for the publications made accessible in the public portal are retained by the authors and/or other copyright owners and it is a condition of accessing publications that users recognise and abide by the legal requirements associated with these rights.

- Users may download and print one copy of any publication from the public portal for the purpose of private study or research.

- You may not further distribute the material or use it for any profit-making activity or commercial gain

- You may freely distribute the URL identifying the publication in the public portal.

If the publication is distributed under the terms of Article 25fa of the Dutch Copyright Act, indicated by the "Taverne" license above, please follow below link for the End User Agreement:

www.tue.nl/taverne

Take down policy

If you believe that this document breaches copyright please contact us at:

openaccess@tue.nl

providing details and we will investigate your claim. 
Eindhoven University of Technology

Department of Mathematics and Computing Science

A Computer Checked Algebraic Verification of a Distributed Summation Algorithm

by

J.F. Groote, F. Monin and J. Springintveld

$97 / 14$

ISSN 0926-4515

All rights reserved

editors: prof.dr. R.C. Backhouse

prof.dr. J.C.M. Baeten

Reports are available at:

http://www.win.tue.nl/win/cs 


\title{
A Computer Checked Algebraic Verification of a Distributed Summation Algorithm
}

\author{
Jan Friso Groote \\ $C W I$ \\ P.O. Box 94079, 1090 GB Amsterdam, The Netherlands \\ $\&$ \\ Department of Mathematics and Computing Science, Eindhoven University of Technology \\ P.O. Box 513, $5600 \mathrm{MB}$ Eindhoven, The Netherlands \\ E-mail: jfg®cri.nl \\ François Monin \\ Department of Mathematics and Computing Science, Eindhoven University of Technology \\ P.O. Box 513, 5600 MB Eindhoven, The Netherlands \\ E-mail: monin@uin.tue.nl \\ Jan Springintveld \\ Computing Science Institute, University of Nijmegen \\ Toernooiveld 1, 6525 ED Nijmegen, The Netherlands \\ E-mail: jans@cs.kun.nl
}

\begin{abstract}
We present an algebraic verification of Segall's Propagation of Information with Feedback (PIF) algorithm and we report on the verification of the proof using the PVS system. This algorithm serves as a nice benchmark for verification exercises (see $[2,18,9])$. The verification is based on the methodology presented in [8] and demonstrates its suitability to deliver mechanically verifiable correctness proofs of highly nondeterministic distributed algorithms.

CR Subject Classification (1991): D.2.4 Program Verification; F.3: Logics and Meanings of Programs.

AMS Subject Classification (1991): 68Q60: Specification and verification of programs; 68Q22: Parallel and distributed algorithms.

Keywords \& Phrases: Distributed Summation Algorithm, Verification, Formal Proof Checking, Process Algebra, $\mu \mathrm{CRL}$, PVS.

Note: The research of the second author is supported by Human Capital Mobility (HCM). The research of the third author is supported by the Netherlands Organization for Scientific Research (NWO) under contract SION 612-33-006. His current affiliation is: CWI, P.O. Box 94079, 1090 GB, Amsterdam, The Netherlands, spring@cwi.nI.
\end{abstract}




\section{Introduction}

The applicability of formal methods for the specification and verification of distributed systems is still a much debated issue. For instance, in [2], Chou claims that there are still no formal methods to reason about distributed systems which are both practical and intuitive. In order to illustrate his opinion he introduces a variant of Segall's PIF (Propagation of Information with Feedback) algorithm [15] which he claims is difficult to prove correct formally. The purpose of this parallel algorithm is to collect the sum of values that are stored by processes which form the nodes of a finite, connected network. The algorithm is indeed an interesting benchmark problem for verification because it is highly parallel and non-deterministic. As such it has been treated in $[2,18,9]$.

Here we present a verification of a distributed summation algorithm in $\mu \mathrm{CRL}$, which is a process algebra which allows processes parameterised with data $[7,6]$. The correctness of the algorithm is stated as a process equation (Theorem 3.5), the proof of which is a straightforward application of the methodology from [8], which is a combination of algebraic and assertional techniques. In [10] it is shown how proofs using this methodology can be proof-checked by computer using the proof checker COQ [3]. Here we have used similar techniques to check the verification using the theorem prover PVS from SRI $[12,13,14,16]$.

This paper is organised as follows. The algorithm is described informally in Section 2 and formally in Section 3. In Section 4, a linear process equation for the algorithm is given and it is proven that the resulting process does not admit infinite sequences of internal actions. Section 5 contains a set of invariants that characterise the reachable states of the algorithm. In Section 6, a state mapping is devised that relates configurations of the implementation to corresponding configurations of the specification. We prove that the state mapping is a branching bisimulation between the implementation and the specification. In section 7 , we report on how we checked the proof in PVS. Section 8 contains a comparison of our proof with three other verifications of the summation algorithm [18, 2, 9]. Finally, Appendix A contains a short overview of the language $\mu \mathrm{CRL}$ and the methodology of [8].

\section{Acknowledgement}

Thanks go to Bas Luttik for carefully proof reading, and to Twan Basten and Jozef Hooman for assistance with PVS.

\section{Description}

The distributed summation algorithm does the following. Consider a set of processes that are connected via some network of bidirectional links (see e.g. Figure 1). We assume that all processes are connected, i.e. from each process we can reach any other process via one or more links. Each process contains some number, not known to other processes. The algorithm describes how to collect all numbers such that one designated (root) process can output the sum of these numbers. The major difficulty in doing so is to use each value in each process exactly once.

The algorithm is described as the parallel composition of a (finite) number of processes, indexed by natural numbers. Each process works in exactly the same way, except for the root 


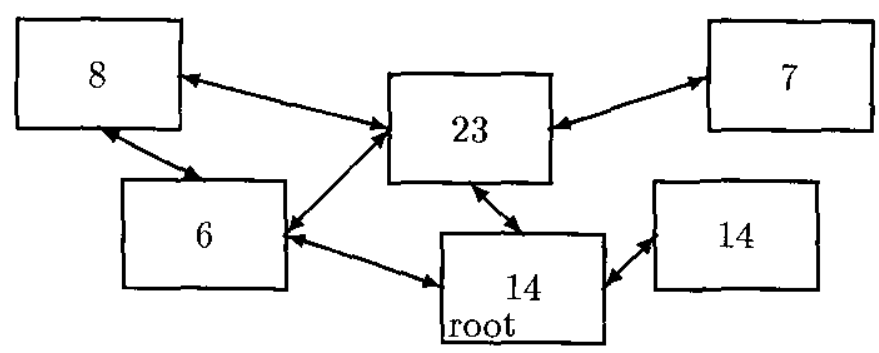

Figure 1: A set of distributed processes

process, which has number 0 . This process differs from the other processes in the sense that initially it is already started, and when it has collected all sums of its neighbours, it issues a $\overline{r e} \bar{p}$ message to indicate the total sum to the outside world, instead of a partial sum to a neighbour.

The overall idea behind the algorithm is that a minimal spanning tree over the links between the processes is constructed with as root the process 0 . All partial sums are then sent via this spanning tree to the root. The difficulty of this protocol is that it is not a priori known how the spanning tree will look like. For every run of the algorithm nondeterministically a different spanning tree may be constructed.

Initially, a process is waiting for a start message from a neighbour. After it has received the first start message, the process is considered part of the spanning tree and the process by which it is started is called its parent. Thereafter it starts all its neighbours except its parent by a start message.

- Those neighbours that were not yet part of the minimal spanning tree will now become part of it with the current process as parent. Eventually, these neighbours will send a partial sum to the current process using an answer message.

- Those neighbours that were already part of the spanning tree ignore the start message. Note however that due to symmetry these processes will also send a start message to the current process.

So, a process gets from each neighbour except its parent either a partial sum or a start message. After having received these messages, it adds all received partial sums to its own value, and sends the result as a partial sum to its parent. Eventually, the root process 0 has received all partial sums, and it can report the total sum.

Theorem 3.5 says that this simple scheme is correct, i.e., if each process is connected to the root, processes do not have themselves as neighbours and the neighbour relation is symmetric, then the distributed summation algorithm computes the sum of the values of the individual processes. Note that if any of the stated conditions on the topology does not hold, the algorithm either deadlocks, not yielding a result, or it does not sum up all values.

\section{Formal specification}

In this section we will formalise the description given above and state the correctness criterion. The algorithm is described as the parallel composition of the algorithms for the individual 
nodes in the network, which are described generically by means of a linear process equation. For a short introduction to the $\mu \mathrm{CRL}$ syntax of processes, we refer to Appendix A.

For the formal specification, we need the data type Bool of the booleans $T$ and $F$ and the usual operators $\wedge, \vee, \rightarrow$ and $\neg$. We also use natural numbers $\mathbb{N}$ with addition and (cut-off) subtraction.

The data type nSet denotes finite sets of natural numbers. For such a set $N$ we let $\operatorname{rem}(i, N)$ represent the set $N$ where element $i$ has been removed. The function size $(N)$ yields the number of different elements in the set. We use $\epsilon$ and $\notin$ to test membership of a set.

We also use lists of natural numbers nList and lists of sets of natural numbers SList. Positions in lists are indexed by natural numbers, starting with index 0 . For a list $l, l[i]$ is the element at position $i$ of the list. We write $l i]:=t$ for the list $l$ where $t$ has been put at position $i$. As these data types are fairly standard, we have omitted their specification using abstract data types.

The processes of the network interact via matching actions st, $\overline{s t}$ (for start), ans, $\overline{a n s}$ (for answer) and the total sum is communicated using a $\bar{r} \overline{e p}$ (for report) action. Although communication is synchronous, we think of the overbarred action as a send activity, and a non-overbarred action as the receiving activity. If an action $a$ synchronises with an action $\bar{a}$, we call the resulting communication $a^{\star}$. In $\mu \mathrm{CRL}$ we formally declare the actions and communications as follows.

act $s t, \overline{s t}, s t^{\star}: \mathbb{N} \times \mathbb{N}$ (parameters: destination, source) ans, $\overline{\text { ans }}$, ans ${ }^{\star}: \mathbb{N} \times \mathbb{N} \times \mathbb{N}$ (parameters: destination, source, value)

$\overline{r e p}: \mathbb{N}$ (parameter: value)

$\operatorname{comm} s t \mid \overline{s t}=s t^{\star}$ ans $\mid \overline{a n s}=a n s^{\star}$

Definition 3.1 (Processes). Processes $P$ are described by means of six parameters:

- $i$ : the ID-number of the process.

- $t$ : the total sum computed so far by the process. Initially, it contains the value that is contributed by process $i$ to the total sum.

- $N$ : a set of neighbours to which the process still needs to send a $\overline{s t}$ message.

- $p$ : the index of the initiator, or parent, of the process. Variable $p$ is also called the parent link of $i$.

- $w$ : The number of st and ans messages that the process is still waiting for.

- $s$ : the state the process is in. The process can be in three states, denoted by $0,1,2$. If $s$ equals 0 , the process is in its initial state. If $s$ equals 1 , the process is active. If $s$ equals 2, the process has finished and behaves as deadlock. 


$$
\begin{aligned}
& P(i, t: \mathbb{N}, N: n S e t, p, w: \mathbb{N}, s: \mathbb{N})= \\
& {[s=0] \Rightarrow \sum_{j: \mathbb{N}} s t(i, j) P(i, t, \operatorname{rem}(j, N), j, \operatorname{size}(N)-1,1)+} \\
& \sum_{j: \mathbb{N}}[j \in N \wedge s=1] \Rightarrow \overline{s t}(j, i) P(i, t, \operatorname{rem}(j, N), p, w, s)+ \\
& \sum_{j, m: \mathbb{N}}[s=1] \Rightarrow \operatorname{ans}(i, j, m) P(i, t+m, N, p, w-1, s)+ \\
& \sum_{j: \mathbb{N}}[s=1] \Rightarrow \operatorname{st}(i, j) P(i, t, N, p, w-1, s)+ \\
& {[i=0 \wedge N=\emptyset \wedge w=0 \wedge s=1] \Rightarrow \overline{\operatorname{rep}}(t) P(i, t, N, p, w, 2)+} \\
& {[i \neq 0 \wedge N=\emptyset \wedge w=0 \wedge s=1] \Rightarrow \overline{a n s}(p, i, t) P(i, t, N, p, w, 2)}
\end{aligned}
$$

In line 1 of $P$ above, process $i$ is in its initial state and an st message is received from some process $j$, upon which $j$ is stored as the parent and $s$ switches from 0 to 1 , indicating that process $i$ has become active. Since it makes no sense to send start messages to one's parent, $j$ is removed from $N$. The counter $w$ is initialised to the number of neighbours of $i$, not counting process $j$. In line 2, a $\overline{s t}$ message is sent to a neighbour $j$, which is thereupon removed from $N$. In line 3 , a sum is received from some process $j$ via an ans message containing the value $m$, which is added to $t$, the total sum computed by process $i$ so far. The counter $w$ is decreased. In line 4 a st message is received from neighbour $j$. The message is ignored, except that the counter $w$ is decreased. In line 5 a $\overline{r e p}(t)$ is sent (in case $i=0$ ), when process 0 is active, there are no more ans or st messages to be received (formalised by the condition $w=0$ ), and a $\overline{s t}$ message has been sent to all neighbours (formalised by the condition $N=\emptyset$ ). The status variable $s$ becomes 2, indicating that process 0 is no longer active. Line 6 is as line 5 but for processes $i \neq 0$; now an $\overline{a n s}$ message is sent to parent $p$, containing the total sum $t$ computed by process $i$.

Next, we define the parallel composition of $n+1$ copies of the process $P$. The result can be viewed as a network of processes in the following way. Think of the $n+1$ nodes of the network as items in a list of length $n+1$. The neighbour relation is given by a list $n$ of length $n+1$ of finite sets of natural numbers, with at each position $i$ the set of neighbours of process $i$. The $t$-values of the processes are put in a list $t$ of length $n+1$ of natural numbers, with at position $i$ the $t$-value of process $i$. Similarly, the lists $p, w, s$ contain the values of the variables $p, w$ and $s$ of all processes, respectively.

Definition 3.2 (Parallel composition of processes).

$$
\begin{aligned}
& \operatorname{Impl}(n: \mathbb{N}, t: n L i s t, \boldsymbol{n}: S L i s t, \boldsymbol{p}: n L i s t, \boldsymbol{w}: n L i s t, \boldsymbol{s}: n L i s t)= \\
& \quad P(0, t[0], \boldsymbol{n}[0], \boldsymbol{p}[0], \boldsymbol{w}[0], s[0]) \triangleleft n=0 \triangleright \\
& \quad(P(n, t[n], \boldsymbol{n}[n], \boldsymbol{p}[n], \boldsymbol{w}[n], \boldsymbol{s}[n]) \| \operatorname{Impl}(n-1, \boldsymbol{t}, \boldsymbol{n}, \boldsymbol{p}, \boldsymbol{w}, \boldsymbol{s}))
\end{aligned}
$$


Next, we formulate some requirements on the topology of the network.

Definition 3.3 (Requirements for topology). We fix a natural number $n$, denoting the number of non-root processes in the network, a list of natural numbers $t_{0}$ of length $n+$ 1 , containing the initial $t$-values of each of the processes, and a list (of length $n+1$ ) of sets of natural numbers $n_{0}$, containing for each process the id's its neighbours. We define goodtopology $\left(n, n_{0}\right)$ as the conjunction of the following properties:

- No process has a link to itself: $\forall i \quad i \notin n_{0}[i]$;

- The neighbour relation is symmetric: $\forall i, j \leq n i \in n_{0}[j] \leftrightarrow j \in n_{0}[i]$;

- Every process $i \leq n$ is connected to process 0 :

for all $i \leq n$ there exist $m \leq n$ and $i=i_{0}, \ldots, i_{m}=0$ such that, for all $0 \leq l<m$, $i_{l+1} \in n_{0}\left[i_{l}\right]$.

- $n_{0}$ only contains valid neighbours: $\forall i \forall j \leq n i \in n_{0}[j] \rightarrow i \leq n$.

Definition 3.4 (Distributed Summing Algorithm). The distributed summation algorithm $D S u m$ is defined as $I m p l$, initialised with, apart from $n, \boldsymbol{t}_{0}$, and $\boldsymbol{n}_{0}$, the following special values:

- $p_{0}$, a list of $n+10$ 's, saying that initially each process considers process 0 as its initiator.

- $\boldsymbol{w}_{0}$, a list of length $n+1$, with at each position $i$ the size of the set $n_{0}[i]$. Thus, initially every process expects a message from all its neighbours.

- $s_{0}$, a list of length $n+1$, with in the first position a 1 , to indicate that process 0 is active, and at the remaining $n$ positions a 0 , to indicate that all other processes are still sleeping.

We leave it to the reader to devise algebraic specifications of these lists. We put

$$
\operatorname{DSum}\left(n, t_{0}, \boldsymbol{n}_{0}\right)=\operatorname{Impl}\left(n, t_{0}, \boldsymbol{n}_{0}, \boldsymbol{p}_{0}, \boldsymbol{w}_{0}, \mathbf{s}_{0}\right)
$$

The theorem below states correctness of the summation algorithm. It says that in a topology as described above, the distributed summation algorithm correctly reports the sum of all values in the processes and halts. The right hand side mentions a function sum, which sums up the numbers in a list of natural numbers.

The remainder of this paper is devoted to proving this theorem; it is repeated and proved as Theorem 6.3.

\section{Theorem 3.5.}




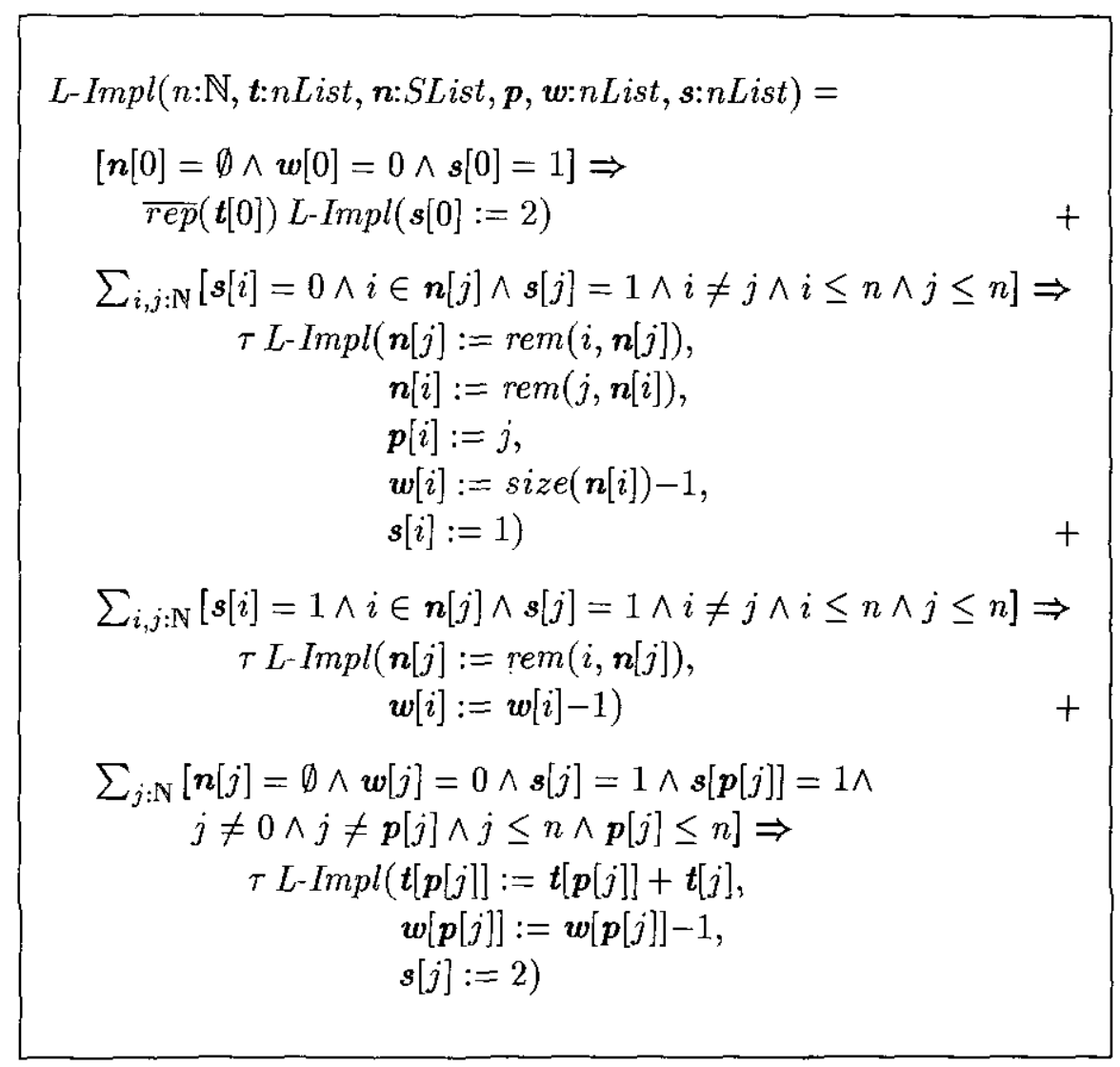

Table 1: Linearisation of the implementation

$$
\operatorname{goodtopology}\left(n, n_{0}\right) \rightarrow \tau \tau_{I} \partial_{H}\left(\operatorname{DSum}\left(n, t_{0}, n_{0}\right)\right)=\tau \overline{r e p}\left(\operatorname{sum}\left(t_{0}\right)\right) \delta
$$

where $I=\left\{s t^{\star}, a n s^{\star}\right\}$ and $H=\{s t, a n s, \overline{s t}, \overline{a n s}\}$. In the trivial case that process 0 has no neighbours, the $\tau$ 's at the left and right hand side of the equation may be omitted.

\section{Linearisation}

In Table 1, we define the process $L$ - Impl, which in Lemma 4.1 is stated to be a convergent linearisation of $\tau_{I} \partial_{H}(\operatorname{Impl}(n, t, n, p, w, s))$. The first and second $\tau$-actions originate from hiding the action $s t^{\star}$. The third $\tau$-action comes from hiding $a n s^{\star}$. In the recursive calls of $L$-Impl only the parameters that are changed are displayed.

\section{Lemma 4.1.}

1. L-Impl in Table 1 is convergent, i.e. does not admit infinite $\tau$-paths.

2. $\tau_{l} \partial_{H}(\operatorname{Impl}(n, \boldsymbol{t}, \boldsymbol{n}, \boldsymbol{p}, \boldsymbol{w}, \boldsymbol{s}))=\operatorname{L-Impl}(n, \boldsymbol{t}, \boldsymbol{n}, \boldsymbol{p}, \boldsymbol{w}, \boldsymbol{s})$. 
3. $\tau_{I} \partial_{H}\left(D S u m\left(n, t_{0}, n_{0}\right)\right)=L-\operatorname{Impl}\left(n, t_{0}, n_{0}, p_{0}, w_{0}, s_{0}\right)$

\section{Proof.}

1. At each $\tau$-step, either a link in $n$ is removed, or a process moves from state 1 to state 2. Hence, the sum of the number of links in $n$ and the number of processes in state 0 or 1 strictly decreases with each $\tau$-step.

2. This follows from Theorem 3.5 in [5] and application of $\tau_{I}$ and $\partial_{H}$.

3. By item 2 .

\section{Invariants}

We provide a number of invariants of which most express that bookkeeping is done properly (see Appendix A for a precise definition of invariants). The most interesting are invariants 14,15 and 16. The first of these three implies that from each process in state 1 process 0 is reachable in a finite number of steps by iteratively following parent links (i.e. following variable $p$ ). As each process has a unique parent, this is an alternative way of saying that the parent links constitute a tree structure with process 0 as root (and a self-loop at the root). Invariant 15 expresses that along each such path all processes are in state 1 too, meaning that they are willing to pass partial results along. Invariant 16 expresses that the total sum in the processes is maintained in the processes that are not in state 2 . We will see that at a certain moment all processes, except process 0 , are in state 2 , which implies that at that moment the total sum is present in process 0 .

The invariants mention the functions Preach, starters, children, and $s u m_{0,1}$, which are defined first.

Definition 5.1. Let $\boldsymbol{t}, \boldsymbol{n}, \boldsymbol{p}, \boldsymbol{s}$ be as in Definition 3.2.

- The function Preach $(i, j, \boldsymbol{p}, m)$ expresses that from process $i$ process $j$ can be reached by following the parent links in $\boldsymbol{p}$. So Preach $(i, j, \boldsymbol{p}, m)$ holds if there exist $i=i_{0}, \ldots, i_{m}=$ $j$ such that, for all $0 \leq l<m, p\left[i_{l}\right]=i_{l+1}$.

- $\operatorname{starters}(i, \boldsymbol{n})$ is the number of sets $L$ in $\boldsymbol{n}$ such that $i \in L$. Intuitively, $\operatorname{starter} s(i, n)$ is the number of processes that still want to send a $\overline{s t}$ message to process $i$.

- $\operatorname{children}(i, \boldsymbol{p}, \boldsymbol{s})$ is the number of processes $j \neq 0$ in the list $p$ such that $p[j]=i$ and $\boldsymbol{s}[j]=1$. That is, $\operatorname{children}(i, p, s)$ is the number of active non-root processes that regard process $i$ as their parent.

- $\operatorname{sum}_{0,1}(\boldsymbol{t}, \boldsymbol{s})$ is the sum of the $t[i]$-values of the processes $i$ that are not yet finished, i.e. such that $s[i]=0$ or $s[i]=1$. 
Theorem 5.2. The following are invariants of $L-\operatorname{Impl}(n, t, n, p, w, s)$. Here the universal quantification over $i$ and $j$ is left implicit. The conjunction of the invariants is written as $\operatorname{Inv}\left(n_{0}, t_{0}, n, t, n, p, w, s\right)$. Note that the initial topology $n_{0}$ and the initial distribution of values $t_{0}$ are part of the invariant, although these are not a parameter of $L$-Impl.

1. $s[i] \leq 2$.

2. $\boldsymbol{p}[i] \leq n$.

3. $i \in \boldsymbol{n}[j] \rightarrow i \leq n$.

4. $i \notin n[i]$.

5. $s[0] \neq 0$.

6. $p[0]=0$.

7. $s[i]=0 \wedge j \in n[i] \rightarrow i \in n[j]$.

8. $\boldsymbol{s}[i]=0 \wedge i \in \boldsymbol{n}[j] \rightarrow j \in \boldsymbol{n}[i]$.

9. $s[i]=0 \rightarrow n[i]=n_{0}[i]$.

10. $s[i]=2 \rightarrow \boldsymbol{w}[i]=0 \wedge \boldsymbol{n}[i]=\emptyset$.

11. If a process $i$ is in state 0 , then it can't be a parent:

$s[i]=0 \rightarrow p[j] \neq i$.

12. $\boldsymbol{s}[i]=0 \rightarrow \boldsymbol{w}[i]=\operatorname{starters}(i, \boldsymbol{n}) \wedge \operatorname{starters}(i, \boldsymbol{n})=\operatorname{size}(\boldsymbol{n}[i]) \wedge \operatorname{children}(\boldsymbol{i}, \boldsymbol{p}, \boldsymbol{s})=0$.

13. For every process $i, w[i]$ records exactly the number of messages that are to be received. These can either be st messages, or ans messages:

$\boldsymbol{w}[i]=\operatorname{starters}(i, \boldsymbol{n})+\operatorname{children}(i, \boldsymbol{p}, \boldsymbol{s})$.

14. From every process $i$ process 0 is reachable via parent links in a finite number of steps: $\exists m \operatorname{Preach}(i, 0, p, m)$.

15. If a process $i$ is in state 1 , then its parent is also in state 1:

$s[i]=1 \rightarrow s[p[i]]=1$.

16. As long as no $\overline{r e p}$ message has been issued by process 0 (i.e. $s[0] \neq 2$ ), the total sum (i.e. $\operatorname{sum}\left(t_{0}\right)$ ) is present in the processes that are in state 0 or 1 :

$s[0] \neq 2 \rightarrow \operatorname{sum}_{0,1}(t, s)=\operatorname{sum}\left(t_{0}\right)$.

Proof. The invariants 1 to 12 are easily checked (invariant 6 uses invariant 5). The invariant 13 uses invariants $4,5,6,8$ and 12 . The invariant 14 uses invariant 11 . The invariant 15 uses invariant 13. The last invariant can be proven on its own. 


\section{State mapping, focus points and final lemma}

In order to apply the methodology from [8], we specify a linear process $L$-Spec describing the specification.

proc $\quad L-\operatorname{Spec}(b:$ Bool $)=[b] \Rightarrow \overline{r e p}\left(\operatorname{sum}\left(t_{0}\right)\right) L-\operatorname{Spec}(\neg b)$

Clearly, $L-\operatorname{Spec}(\mathrm{T})=\overline{\mathrm{r}} \overline{\mathrm{e}}\left(\operatorname{sum}\left(\boldsymbol{t}_{0}\right)\right) \delta$.

Furthermore, we provide a state mapping $h$, that specifies how the control variable $b$ of the specification $L$-Spec is constructed out of the parameters $n, t, n, p, w, s$ of the implementation L-Impl. We put

$$
h(n, t, n, p, w, s)=(s[0]=1) .
$$

The intuition behind this definition is as follows. In a configuration $s$ of $L$-Impl that satisfies $s[0]=1, h(s)$ is $T$ (true), so $L$-Spec can perform the $\overline{r e p}$-action, after which it halts. $L$-Impl may not be able to perform a matching $\overline{r e p}$ action directly, since the computation of the value to be reported has not yet finished (i.e., $n[0] \neq \emptyset$ or $\boldsymbol{w}[0] \neq 0$ ). However, using the fact that $L$ - Impl is convergent, we see that after a finite number of internal $\tau$-steps a configuration $s^{\prime}$ is reached where no $\tau$-step is enabled, $s[0]$ is still 1 ( $h$ will be invariant under the $\tau$-steps), but also $n[0]=\emptyset$ and $\boldsymbol{w}[0]=0$. So the $\bar{r} \overline{e p}$-action can be performed (with the correct value), after which $L$-Impl halts. Conversely, it is easy to verify that if in configuration $s L$-Impl can perform the $\overline{r e p}$ action, then $s[0]=1$, so in configuration $h(s)$ the control variable $b=h(s)$ of $L$-Spec has the value $\mathrm{T}$ and the specification $L$-Spec can perform the $\overline{r e p}$-action (with corresponding value). From these observations it will follow that $h$ is indeed a branching bisimulation function.

We formalise this intuitive argument, using focus condition, which is a formula that characterises the configurations of $\mathrm{L}$ - $\mathrm{Impl}$ in which no $\tau$-step is enabled. (These configurations are so-called focus points). Such a formula is extracted from the equation characterising $L-I m p l$ (see Table 1) by negating the guards that enable $\tau$-steps in $L$-Impl. As an optimisation, we have put the first two negated guards together, and have restricted the focus condition to configurations satisfying the invariant.

$$
\begin{aligned}
& F C(n, \boldsymbol{t}, \boldsymbol{n}, \boldsymbol{p}, \boldsymbol{w}, \boldsymbol{s})=\forall i, j \leq n \\
& \quad(\boldsymbol{s}[i]=2 \vee i \notin \boldsymbol{n}[j] \vee s[j] \neq 1 \vee i=j) \wedge \\
& \quad(\boldsymbol{n}[j] \neq \emptyset \vee \boldsymbol{w}[j]>0 \vee s[j] \neq 1 \vee s[p[j]] \neq 1 \vee j=0)
\end{aligned}
$$

We distinguish two kinds of focus points of the distributed summation algorithm. One is the set of configurations where the algorithm has reported the sum and is terminated, so $s[0]=2$. The other one contains the configuration $s^{\prime}$ mentioned above and is characterised by $s[0]=1$. At that moment the correct sum should be reported. Items 1 and 2 of the lemma below say that all conditions in the process $L$-Impl for issuing a $\overline{r e p}$ action are satisfied; so reporting is possible. Item 3 says that in such a case, all other processes are in state 2 . Hence, using invariant 16 (i.e., $s[0] \neq 2 \rightarrow \operatorname{sum}_{0,1}(t, s)=\operatorname{sum}\left(\boldsymbol{t}_{0}\right)$ ) we may conclude that the total sum is indeed collected in process 0 , i.e. process 0 reports the correct sum.

Lemma 6.1. Inv $\left(\boldsymbol{n}_{0}, \boldsymbol{t}_{0}, n, \boldsymbol{t}, \boldsymbol{n}, \boldsymbol{p}, \boldsymbol{w}, \boldsymbol{s}\right)$ and $\boldsymbol{s}[0]=1$ together imply

1. $F C(n, t, n, p, w, s) \wedge s[i]=1 \rightarrow n[i]=\emptyset$ 
2. $F C(n, \boldsymbol{t}, \boldsymbol{n}, \boldsymbol{p}, \boldsymbol{w}, \boldsymbol{s}) \rightarrow \boldsymbol{w}[0]=0$.

3. goodtopology $\left(n, n_{0}\right) \wedge w[0]=0 \wedge i \neq 0 \rightarrow s[i]=2$.

\section{Proof.}

1. Towards a contradiction, assume there exists a process $i$ such that $s[i]=1$ and $n[i] \neq \emptyset$, say $j \in \boldsymbol{n}[i]$. By invariant 4 we have $j \neq i$. By the first part of $F C(n, t, n, p, w, s)$, $s[j]=2$. By invariant $10, w[j]=0$, contradicting invariant 13 (remember that $j \in \boldsymbol{n}[i]$ ).

2. In order to derive a contradiction, assume that $\boldsymbol{w}[0]>0$. For arbitrary $m$, we construct a sequence of $m+1$ processes $0=i_{0}, i_{1}, \ldots, i_{m}$ such that for all $0 \leq l \leq m$, we have $s\left[i_{l}\right]=1, w\left[i_{l}\right]>0, p\left[i_{l+1}\right]=i_{l}$, and if $l \neq 0, i_{l} \neq 0$. Clearly, if $m>n$, there is one element $i_{k} \neq 0$ which appears twice in the path (pigeon hole principle). Hence we get in the path a cycle starting from $i_{k}$ where 0 is not there. So, $i_{0}$ can't be reachable via parent links from $i_{k}$ and in particular from $i_{m}$, this contradicts the existence of the current sequence.

Let a process $i_{l}$ be given such that $\boldsymbol{w}\left[i_{l}\right]>0$ and $s\left[i_{l}\right]=1$. According to invariant 13 at least one of the following should hold.

- There exists some $i$ such that $i_{l} \in n[i]$. By invariant $4, i_{l} \neq i$. By the first part of $F C(n, t, n, p, w, s)$ it follows that $s[i] \neq 1$. So, either $s[i]=2$, but this leads to a contradiction using invariant 10 (remember that $n[i] \neq \emptyset$ ). Or, $\boldsymbol{s}[i]=0$. By invariant $7, i \in \boldsymbol{n}\left[i_{i}\right]$. So, by $F C(n, t, n, p, w, s), s\left[i_{l}\right] \neq 1$. Contradiction.

- Or there is some $i$ such that $p[i]=i_{l}, i \neq 0$ and $s[i]=1$. By the second part of $F C(n, t, n, p, w, s)$, we have $\boldsymbol{w}[i]>0 \vee \boldsymbol{n}[i] \neq \emptyset$. By item 1 of this lemma, $\boldsymbol{n}[i]=\emptyset$. So $\boldsymbol{w}[i]>0$. We can take $i_{l+1}=i$.

3. First, assume there is some process $i \neq 0$ such that $s[i]=1$. Using invariants 13,15 and 14 , it follows that there is a sequence of processes $i=i_{0}, \ldots, i_{m}=0$ such that, for all $0 \leq l<m, i_{l} \neq 0$ (even if it means to cut the path), $p\left[i_{l}\right]=i_{l+1}, s\left[i_{l}\right]=1$ and $\boldsymbol{w}\left[i_{l+1}\right]>0$. In particular $\boldsymbol{w}[0]>0$ contradicting an assumption.

So, assume that there is no process $i \neq 0$ such that $s[i]=1$, but there is some process $i \neq 0$ such that $s[i]=0$. From the topology requirement it follows that there is a sequence $i=i_{0}, \ldots, i_{m}=0$ such that for all $0 \leq l<m, i_{l+1} \in n_{0}\left[i_{i}\right]$. We show that $s\left[i_{l}\right]=0$ for all $l, 0 \leq l \leq m$. This contradicts the assumption that $s[0]=1$.

Note that by assumption $s\left[i_{0}\right]=0$. So let $i_{l}$ such that $s\left[i_{l}\right]=0$. By invariant 9 , it follows that $i_{l+1} \in \boldsymbol{n}\left[i_{l}\right]$. By invariant $13, \boldsymbol{w}\left[i_{l+1}\right]>0$, so $i_{l+1} \neq 0$ and, by invariant $10, s\left[i_{l+1}\right] \neq 2$. As we have excluded that process $i_{l+1}$ is in state 1 , it must hold that $s\left[i_{l+1}\right]=0$, as required.

Below we copy the General Equality Theorem (see Theorem A.3) instantiated for the distributed summation algorithm. It says that, given the invariant, implementation $L$-Impl 
and specification $L$-Spec are equivalent (with or without a preceding $\tau$-step, depending on whether the focus condition holds). Its proof requires that 6 groups of requirements, the socalled matching criteria, are checked. Given Lemma 6.1 this is completely straightforward.

Lemma 6.2. Assume goodtopology $\left(n, n_{0}\right)$.

$$
\begin{array}{ccl}
\operatorname{Inv}\left(\boldsymbol{n}_{0}, t_{0}, n, \boldsymbol{t}, \boldsymbol{n}, \boldsymbol{p}, \boldsymbol{w}, \boldsymbol{s}\right) \rightarrow & & \\
L-\operatorname{Impl}(n, \boldsymbol{t}, \boldsymbol{n}, \boldsymbol{p}, \boldsymbol{w}, \boldsymbol{s}) & \triangleleft F C(n, \boldsymbol{t}, \boldsymbol{n}, \boldsymbol{p}, \boldsymbol{w}, \boldsymbol{s}) \triangleright & \tau L-\operatorname{Impl}(n, \boldsymbol{t}, \boldsymbol{n}, \boldsymbol{p}, \boldsymbol{w}, \boldsymbol{s}) \\
\operatorname{L-Spec}(\boldsymbol{s}[0]=1) & \triangleleft F C(n, \boldsymbol{t}, \boldsymbol{n}, \boldsymbol{p}, \boldsymbol{w}, \boldsymbol{s}) \triangleright & \tau L-\operatorname{Spec}(\boldsymbol{s}[0]=1)
\end{array}
$$

Proof. According to [8] it suffices to check that the following instances of the matching criteria are implied by the invariant.

1. By Lemma 4.1.1 L-Impl is convergent.

2. The following three requirements ensure that the state mapping $h$ is invariant under $\tau$-steps of $L$ - Impl.

(a) $s[i]=0 \wedge i \in n[j] \wedge s[j]=1 \wedge i \neq j \wedge i \leq n \wedge j \leq n$ implies $s[0]=(s[i]:=1)[0]$ (note that $(s[i]:=1)[0]$ is the first element of $s$ where the $i^{\text {th }}$ element has been replaced by 1 ).

We distinguish two cases. If $i \neq 0$, the condition trivially holds because in that case $(s[i]:=1)[0]=s[0]$. If $i=0$, one conjunct of the precondition says $s[0]=0$. This contradicts invariant 5 .

(b) $s[i]=1 \wedge i \in n[j] \wedge s[j]=1 \wedge i \neq j \wedge i \leq n \wedge j \leq n$ implies $s[0]=s[0]$.

This requirement clearly holds.

(c) $n[j]=\emptyset \wedge \boldsymbol{w}[j]=0 \wedge \boldsymbol{s}[j]=1 \wedge \boldsymbol{s}[\boldsymbol{p}[j]]=1 \wedge j \neq 0 \wedge j \neq \boldsymbol{p}[j] \wedge j \leq n \wedge \boldsymbol{p}[j] \leq n$ implies $s[0]=(s[j]:=2)[0]$.

This requirement is also trivially valid, because the assumption explicitly says $j \neq 0$. Hence, $(s[j]:=2)[0]=s[0]$.

3. Next, we verify that when the $\overline{r e p}$ action is enabled in $L$-Impl, it is enabled in $L$-Spec: $n[0]=\emptyset \wedge w[0]=0 \wedge s[0]=1$ implies $s[0]=1$. This is obviously true.

4. We must show that if $L$ - Impl is in a focus point (no internal actions enabled) and $L$-Spec can perform a $\overline{r e p}$-action, $L$-Impl can also perform the $\overline{r e p}$ action:

$F C(n, t, n, p, w, s) \wedge s[0]=1$ implies $n[0]=\emptyset \wedge w[0]=0 \wedge s[0]=1$. This is a direct consequence of Lemma 6.1.2 and Lemma 6.1.1.

5. We must show that if the $\overline{r e p}$ action is enabled in $L-I m p l$ then the reported sum is equal to the sum reported in $L$-Spec: $n[0]=\emptyset \wedge w[0]=0 \wedge s[0]=1$ implies $t[0]=\operatorname{sum}\left(t_{0}\right)$. By invariant 16 we have $\operatorname{sum}\left(\boldsymbol{t}_{0}\right)=\operatorname{sum}_{0,1}(\boldsymbol{t}, \boldsymbol{s})$. By definition, $\operatorname{sum}_{0,1}(\boldsymbol{t}, \boldsymbol{s})$ contains the sum of the $t[i]$ values of all processès $i$ that are not in state 2. By Lemma 6.1.3, only process 0 is not in state 2 . Hence $\operatorname{sum}\left(t_{0}\right)=\operatorname{sum}_{0,1}(t, s)=t[0]$. 
6. Finally, we have to show that the $h$-mapping commutes with the $\overline{r e p}$ action, i.e. $(s[0]:=$ $2)[0] \neq 1$. This is easily seen to hold.

$\otimes$

Theorem 6.3.

$$
\text { goodtopology }\left(n, \boldsymbol{n}_{0}\right) \rightarrow \tau \tau_{I} \partial_{H}\left(\operatorname{DSum}\left(n, \boldsymbol{t}_{0}, \boldsymbol{n}_{0}\right)\right)=\tau \bar{r} \overline{\operatorname{ep}}\left(\operatorname{sum}\left(\boldsymbol{t}_{0}\right)\right) \delta
$$

where $I=\left\{s t^{\star}, a n s^{\star}\right\}$ and $H=\{s t, a n s, \overline{s t}, \overline{a n s}\}$. In the trivial case that process 0 has no neighbours, the $\tau$ 's at the left and right side of the equation may be omitted.

Proof. Apply Lemma 6.2 with $t_{0}$ substituted for $t, n_{0}$ for $n, p_{0}$ for $p, w_{0}$ for $\boldsymbol{w}$ and $\boldsymbol{s}_{0}$ for $s$. This substitution reduces the invariant to T. Furthermore, reduction of the term $F C\left(n, t_{0}, n_{0}, p_{0}, w_{0}, s_{0}\right)$ leads to $\forall i i \notin n_{0}[0]$. Thus we have

$$
\begin{array}{cc}
L-\operatorname{Impl}\left(n, t_{0}, \boldsymbol{n}_{0}, \boldsymbol{p}_{0}, \boldsymbol{w}_{0}, \boldsymbol{s}_{0}\right) & \triangleleft \forall i i \notin n_{0}[0] \triangleright \quad \tau L-\operatorname{Impl}\left(n, \boldsymbol{t}_{0}, \boldsymbol{n}_{0}, \boldsymbol{p}_{0}, \boldsymbol{w}_{0}, \boldsymbol{s}_{0}\right) \\
& = \\
L-\operatorname{Spec}(\mathrm{T}) & \triangleleft \forall i i \notin n_{0}[0] \triangleright \quad \tau L-\operatorname{Spec}(\mathrm{T}) .
\end{array}
$$

Hence we can conclude

$$
\tau L-\operatorname{Impl}\left(n, t_{0}, n_{0}, p_{0}, w_{0}, s_{0}\right)=\tau L-\operatorname{Spec}(\mathrm{T})
$$

by adding an initial $\tau$ if appropriate. We can conclude the stronger

$$
L-\operatorname{Impl}\left(n, \boldsymbol{t}_{0}, \boldsymbol{n}_{0}, \boldsymbol{p}_{0}, \boldsymbol{w}_{0}, \boldsymbol{s}_{0}\right)=L-\operatorname{Spec}(\mathrm{T})
$$

in case $\forall i \quad i \notin n_{0}[0]$, i.e. in case process 0 has no neighbours.

By Lemma 4.1.3, we have $\tau_{l} \partial_{H}\left(\operatorname{DSum}\left(n, \boldsymbol{t}_{0}, \boldsymbol{n}_{0}\right)\right)=L-\operatorname{Impl}\left(n, \boldsymbol{t}_{0}, \boldsymbol{n}_{0}, \boldsymbol{p}_{0}, \boldsymbol{w}_{0}, \boldsymbol{s}_{0}\right)$. We also have seen that $L-\operatorname{Spec}(\mathrm{T})=\overline{r e} \bar{p}\left(\operatorname{sum}\left(\boldsymbol{t}_{0}\right)\right) \delta$. The theorem follows.

\section{Computer-checking the verification}

The proof of Theorem 6.3 establishing the correctness of the distributed summation algorithm (DSA for short) has been computer checked with the theorem prover PVS (2.1 Test (patch level 2.399)).

We have first defined in PVS the general notion of linear process equations (LPEs), and formulated the General Equality Theorem A.3 which allows to prove equality between processes specified by LPEs (see Appendix A). Using this theorem we have given a complete formalization of the proof in PVS. We have not mechanically checked the proof of GET itself since it is part of the logical framework of $\mu \mathrm{CRL}$ and we therefore considered it as given for the verification of this particular distributed algorithm. Also, the linearisation of the protocol, i.e. Lemma 4.1.2, was not checked. We note that linearisation can be done mechanically [5]. The whole of the definitions, lemmas and proof-scripts can be obtained by mailing one of the authors. 
The specification language of PVS is a higher-order typed logic $([13,14,16])$, with many built-in types including booleans, integers, sequences, lists, etc. For example, upto(i): TYPE $=\{s:$ nat $\mid s<=i\}$ is the subtype of the integers less or equal to $i$. New types may be added together with functions, tuples, records, predicate subtypes, abstract datatypes. Usually, a PVS specification consist of one or several theories. A theory can have parameters and can be imported by other theories (see [13]).

In the vernacular of PVS, the complete main theorem (Theorem 6.3), including the note on the "trivial case", is represented as follows (in order to make clear what the PVS code is, we typeset it in teletype font):

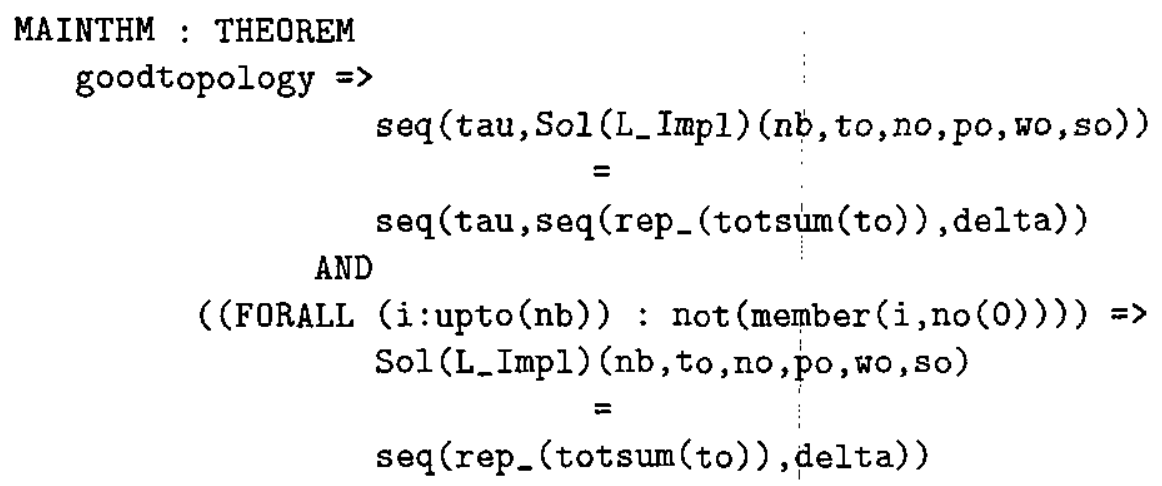

where seq, rep, tau, delta represent respectively the sequential composition operator $\overline{r e p}, \tau$ and $\delta$. Sol (L_Impl) is the solution of the linear process equation $L$-Impl depicted in Table 1. The value $\mathrm{nb}$ is the number $n$ of non-root processes in the network. The terms to, no,po, wo, so stand respectively for the initial values $t_{0}, n_{0}, p_{0}, w_{0}, s_{0}$, while totsum(to) stands for the sum of the values in $t_{0}$. Finally, goodtopology correspond to the topology requirements goodtopology $\left(n, n_{0}\right)$. The values $\mathrm{nb}$ and $\left(t_{0}, n_{0}, \boldsymbol{p}_{0}, \boldsymbol{w}_{0}, s_{0}\right)$ have been introduced as constants in PVS and therefore do not appear in goodtopology.

In the following subsections we describe the formalisation of the proof of MAINTHM in PVS. First, we describe how the General Equality Theorem has been encoded. In subsection 7.2, the data of $L$-Impl and $L$-Spec, the initial values $t_{0}, n_{0}, \boldsymbol{p}_{0}, \boldsymbol{w}_{0}, \boldsymbol{s}_{0}$, and the topology are given. In the next subsection we show how the invariant property, i.e. Theorem 5.2 , has been proven in PVS. In subsection 7.4 the proof of Lemma $6: 1$ is described. In the following subsection, we present the formalisation of the state mapping, the focus points and the matching criteria. We conclude the proof in subsection 7.6. Finally, in subsection 7.7 , we discuss the formalisation in PVS.

\subsection{The General Equality Theorem}

We have devised the general notion of a linear process equation (LPE) depending on a. data type D as a parameter in a theory LPES [D :TYPE] : THEORY (see Definition A.1 in Appendix A). This theory imports the theory THEDATA: THEORY which specifies the processes, actions and domains over which summation takes place in the definition of the LPEs. The set of LPEs has been defined as a type LPE: TYPE $\mid=\ldots$ Each element of this type corresponds to a linear process equation. Theorem A.3 mentions two LPEs of which the second one runs 
over a set of actions from which $\tau$ has been removed. So, in the same theory, we have defined a subtype ALPE: TYPE $=\ldots$ of the previous type, containing elements that are LPEs but from which the $\tau$ action has been removed. We do not provide the types LPE and ALPE here because their definition is somewhat unwieldy and not necessary to understand the main steps of the verification.

Then, in a new theory THGET [DX,DY:TYPE]: THEORY, Theorem A.3 has been introduced. Here the data types DX and DY are parameters of the theory THGET that can be instantiated with data types. Since the LPEs involved rely on different data types, the theory imports both LPES [DX] and LPES[DY]. This is actually the way in PVS to use polymorphic types. The invariant property, the focus points and the criteria occurring in Theorem A.3 have been translated into the theory as predicates. Theorem A.3 is then represented as an axiom as follows:

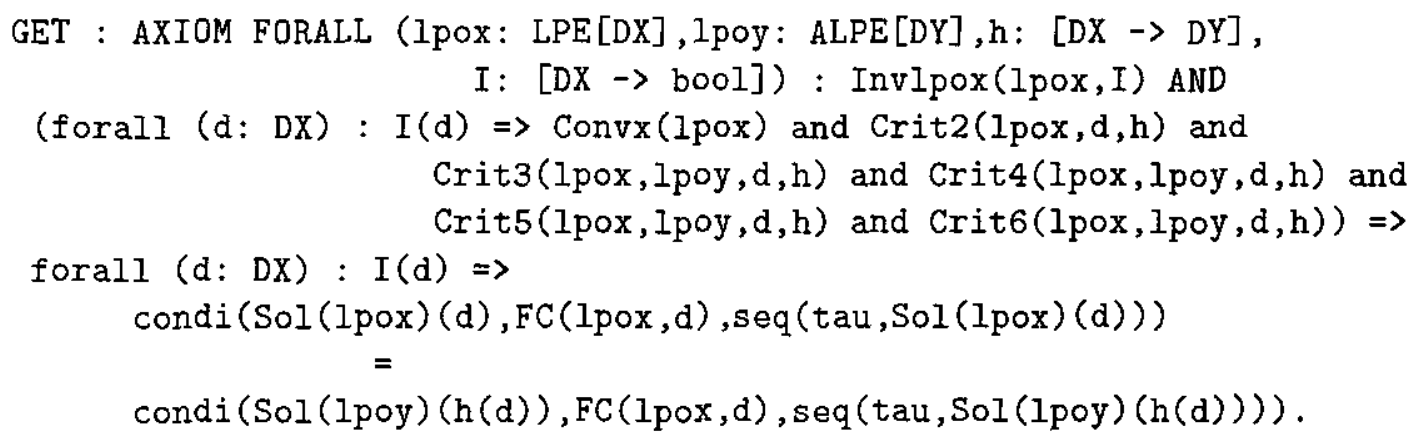

Here, condi ( $\arg 1, \arg 2, \arg 3$ ) denotes the conditional construct arg1 $\triangleleft$ arg2 $\triangleright$ arg3.

The invariant property Invlpox(lpox:LPE [DX], I: [DX $\rightarrow$ bool]) asserts that the function $I:[D X \rightarrow$ bool] ) is an invariant of Ipox, that is to say, for any state d:DX, if I (d) holds and a step can be performed by lpox, then I holds in the new state. The focus condition FC (lpox: $L P E[D X], d: D X)$ characterises the states $d$ of the LPE implementation Ipox in which no $\tau$-action is enabled. The first criterion Convx (lpox:LPE[DX]) says that the LPE implementation lpox must be convergent. Crit2 (Ipox:LPE $[D X], d: D X, h:[D X \rightarrow D Y]$ ) says that if in a state $d$ in the LPE implementation lpox, an internal step can be done, then this internal step is not observable modulo the state mapping $h$. Crit3 (Ipox:LPE [DX], lpoy:LPE [DY], d: $D X, h:[D X \rightarrow D Y]$ ) says that when the LPE implementation lpox can perform an external step according to the value of $d$, then the corresponding point (modulo $h$ ) in the LPE specification lpoy must also be able to perform this step. Crit4(lpox:LPE[DX], lpoy:LPE[DY],d: $D X, h:[D X \rightarrow D Y])$ says that in a focus point FC(lpox ,d) of the LPE implementation lpox, an action can be performed if it is enabled in the LPE specification lpoy. Crit5 (lpox, lpoy, $d, h$ ) and Crit6 (lpox, lpoy, $d, h$ ) express that corresponding external actions carry the same data parameter (modulo $d$ and $h$ ) and lead to corresponding states.

In order to define $L$-Impl and $L$-Spec, data types DX and DY corresponding to their parameters have been made explicit in a theory IMPL: THEORY. The theory IMPL imports the theories LPES [DX] and LPES [DY]. The distributed summation algorithm $L-I m p l$ has been defined as an LPE by L_Impl: $\quad$ LPE $[D X]=\ldots$ corresponding to the formalisation of $L$-Impl depicted in Table 1. In the same way the linear process $L-S p e c$ described in Section 6 has been defined 
to be of type ALPE, L_Spec: ALPE $[D Y]=\ldots$.

The various parts of the proof of MAINTHM are provided in the theories DSA1, DSA2, DSA3, DSA4 and DSA: THEORY.

\subsection{The data types, the initial values and the topology}

Below we give the data types DX and DY corresponding to the types of the parameters of $L$-Impl and L-Spec. Since PVS allows one to have bounded types using subtypes, we have used families indexed by the finite set of processes in a network. Here nb denotes the number of non-root processes, it has been introduced in the THEDATA theory:

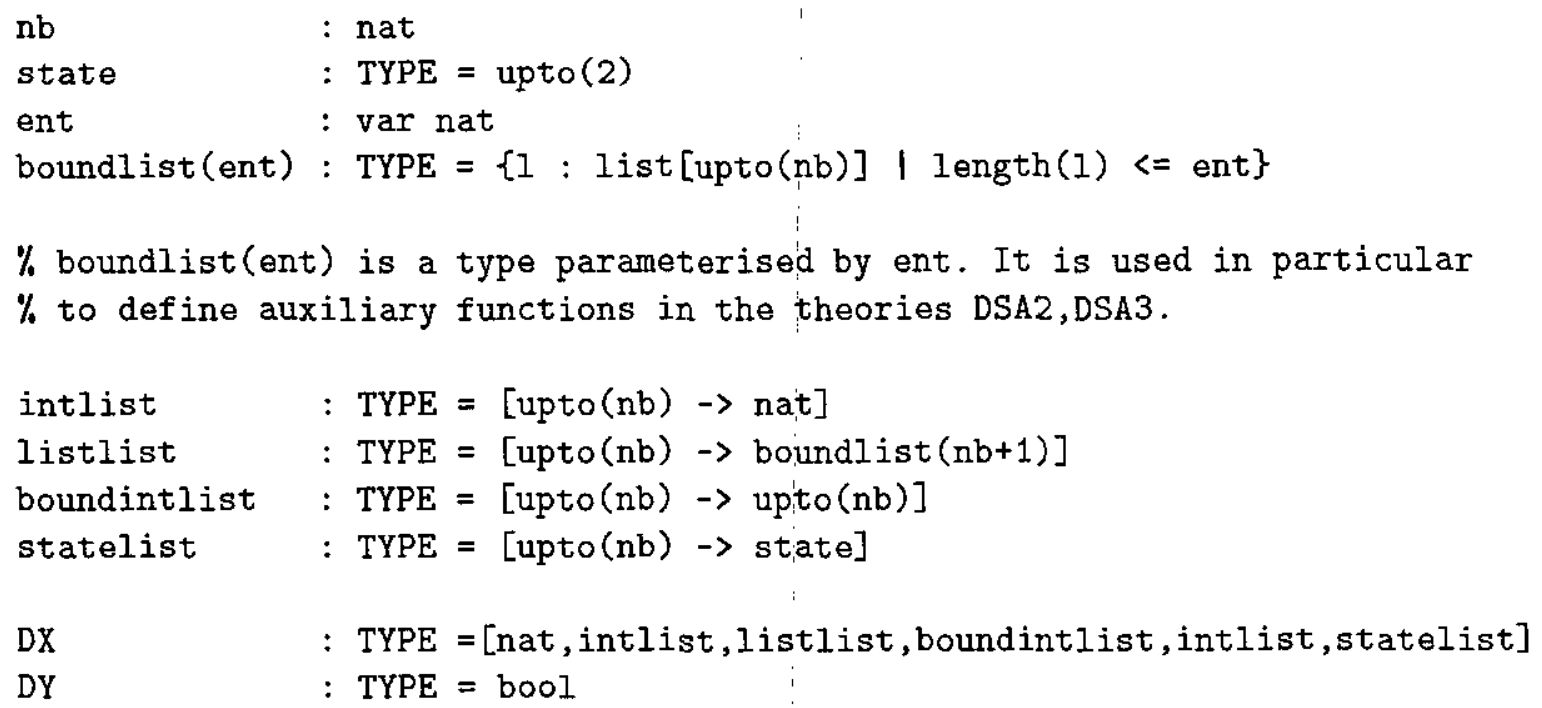

The initial values $t_{0}, n_{0}, p_{0}, w_{0}, s_{0}$ of Definition 3.4 appear in the IMPL theory as follows:

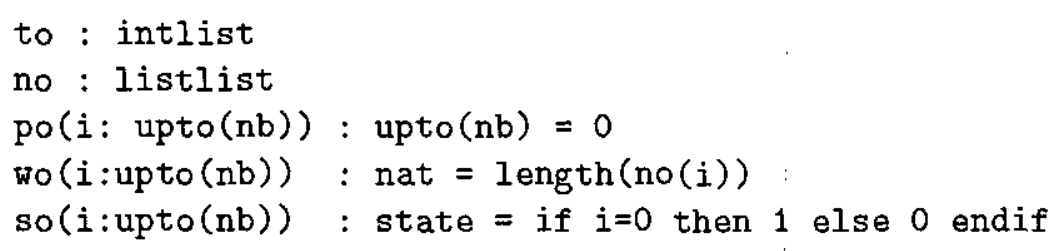

Here, e.g., the domain and range of wo are respectively upto(nb) and nat, so the type of wo is intlist. For each element $i$ of type upto(nb), wo( $i$ ) is equal to length(no(i)). This corresponds to the fact that $w_{0}$ is a list of length $n+1$, with at each position $i$ the size of the set $n_{0}[i]$. Likewise, the domain and range of so are respectively upto $(\mathrm{nb})$ and (a subset of) nat, hence the type of so is statelist. Also, po is the null function of type boundintlist.

The definition of boundintlist implies the fourth property of the topology in Definition 3.3. So it is not necessary to introduce it into goodtopology. On the other hand, we modified the topology with a new requirement TOP4 asserting that each element has at most one occurrence in $n_{0}[j]$ (neighbours). This is obviously true for sets but wrong for lists. We could also have used sets for neighbours, as used in the previous sections, but it is more convenient using lists together with this requirement. The requirement TOP4 allows us to have the following properties: 
Member_three : LEMMA FORALL (i: upto(nb), l: boundlist $(n b+1)$ ) : $\operatorname{not}(\operatorname{member}(i, \operatorname{rem}(i, 1)))$

Lightlist three : LEMMA FORALL ( $i$ : upto(nb), I: boundlist $(n b+1)$ ) : nodouble $(1)$ and member $(i, 1) \Rightarrow$ length $(1)=1+\operatorname{length}(\operatorname{rem}(i, 1))$

where rem removes all occurrences of $i$ in 1 . The non-redundant property TOP4 is necessary to prove that the invariant predicate Inv applied to the initial values holds (for Inv, see subsection 7.3):

Initialinv : LEMMA goodtopology $\Rightarrow \operatorname{Inv}(\mathrm{nb}, \mathrm{to}, \mathrm{no}, \mathrm{po}$, wo, so)

where below goodtopology is defined:

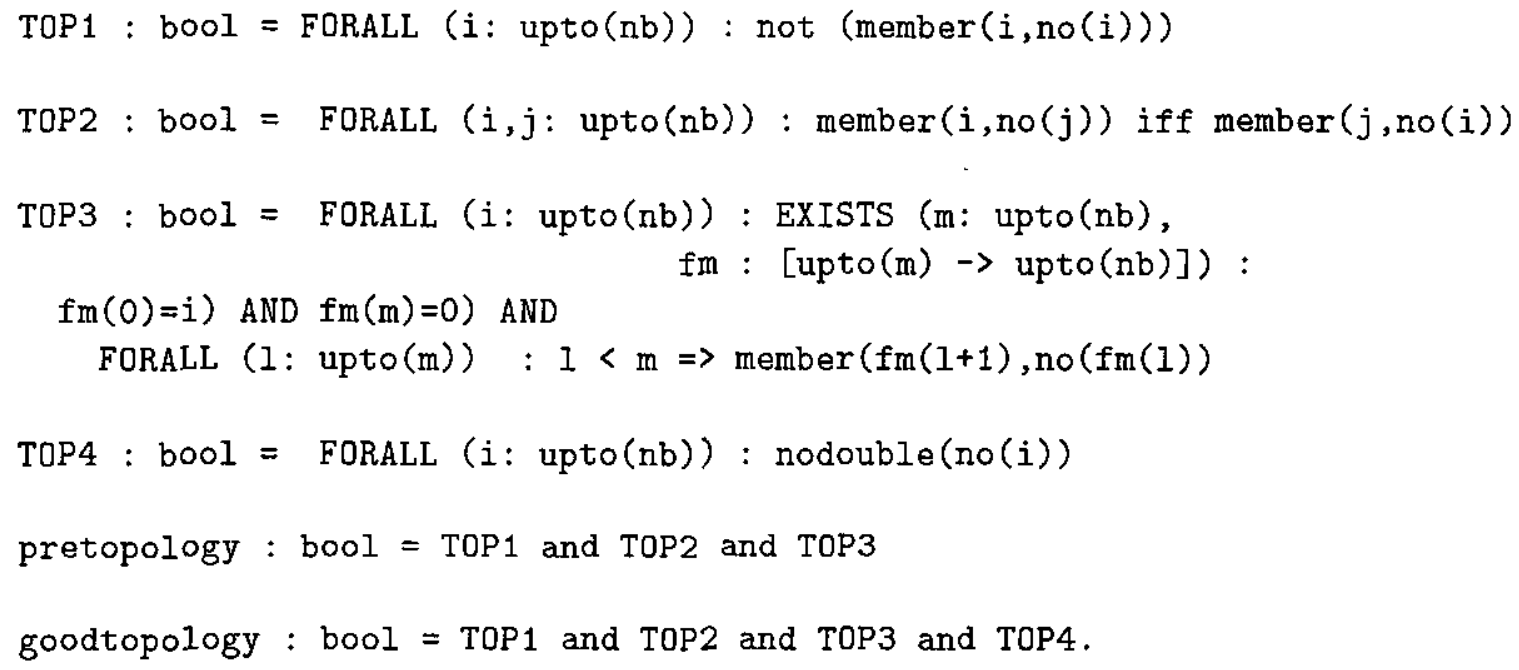

\subsection{The invariant property}

The use of the GET theorem requires an invariant property, that is to say the existence of a function $I:[D X \rightarrow$ bool] such that in particular the predicate Invlpox (L_Impl, I) holds. For I we provide a function Inv which corresponds to the formalisation of the conjunction of the items in Theorem 5.2. Actually, the first three items of Theorem 5.2 are not included in Inv, as they are direct consequences of the definitions of statelist, boundintlist and listlist, respectively. In the proof of MAINTHM, the predicate Invlpox (L.Impl, Inv) leads to the requirement to prove the four predicates S1Inv, S2Inv, S3Inv and S4Inv, each one corresponding to a summand of the LPE L-Impl in Table 1. The predicate S1Inv corresponds to the summand with the $\overline{r e p}$ action, the remaining three predicates correspond to the summands with $\tau$ actions.

S1Inv: LEMMA FORALL ( $\mathrm{k}$ : nat, $\mathrm{t}$ : intlist, $\mathrm{n}$ : listlist,p: boundintlist,

w: intlist, $\mathrm{s}$ : statelist) :

$\operatorname{Inv}(k, t, n, p, w, s)$ AND $n(0)=n u l l$ and $w(0)=0$ and $s(0)=1 \Rightarrow$

$\operatorname{Inv}(k, t, n, p, w, s$ with $[(0):=2])$ 


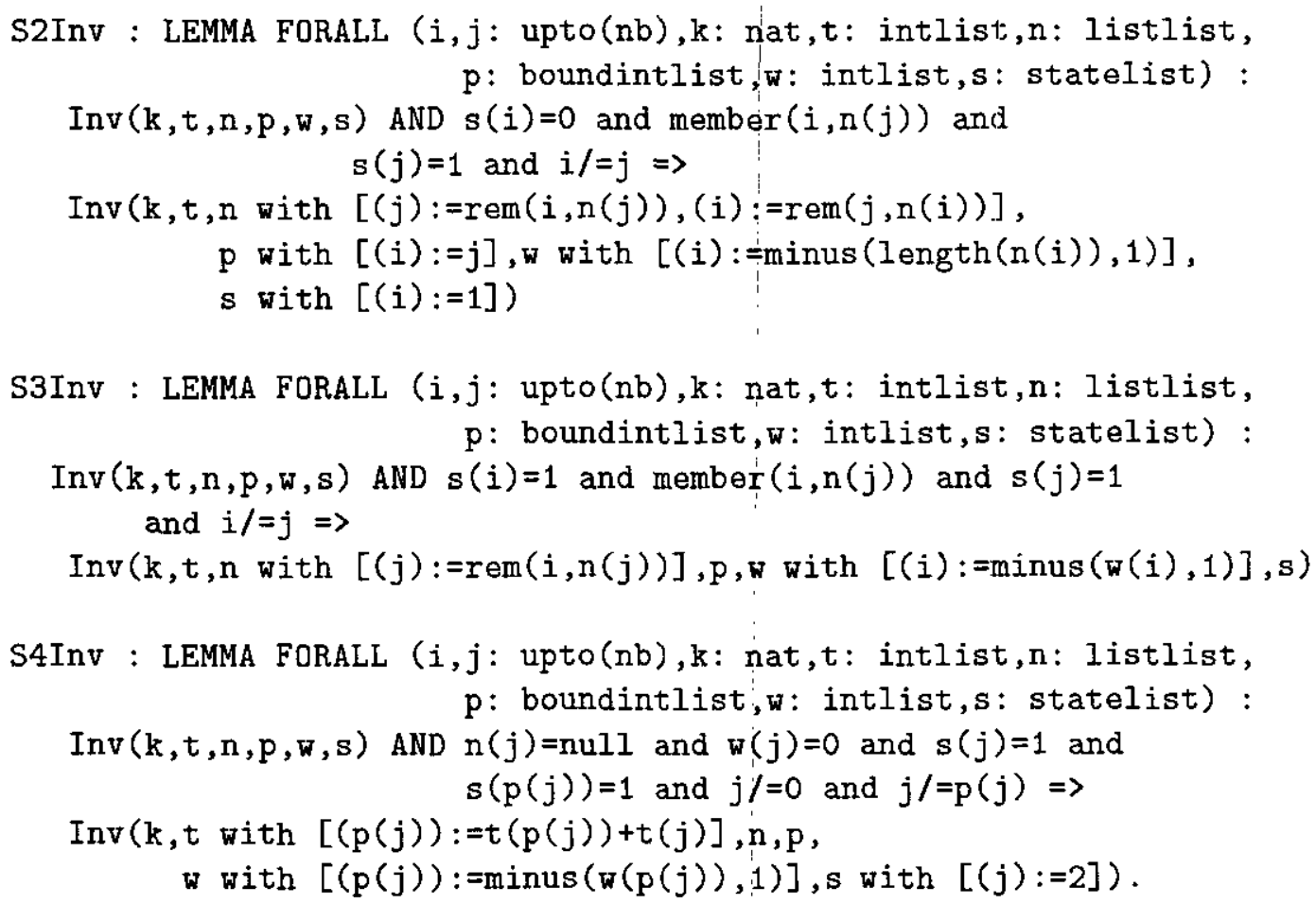

Each of the previous lemmas has been proven in the following way. First, we define predicates Inv4, .., Inv16 corresponding to the items of Theorem 5.2. For example the last predicate is:

Inv16( $\mathrm{k}$ :nat, $\mathrm{t}$ : intlist, $\mathrm{n}:$ listlist, $\mathrm{p}$ : boundintlist, $\mathrm{w}:$ intlist, $\mathrm{s}:$ statelist) : bool $=s(0) /=2 \Rightarrow \operatorname{sum} 0$ and $1(t, s)=t_{\text {ot }} \operatorname{sum}\left(t_{0}\right)$

where sum0and1 $(t, s)$ represents $\operatorname{sum}_{0,1}(t, s)$ (Definition 5.1).

Secondly, for $i=4, \ldots, 16$, we introduced and proved lemmas S1Invi, S2Invi, S3Invi, and S4Inv $i$ (Inv is changed to Invi). As a detail, we mention that items 5 and 6 have been directly put into Inv13, as they are only necessary for the item 13 and easily checked. They obviously still appear in Inv15 because the proofs of $S 1 \operatorname{Inv} 15, \ldots, S 4 \operatorname{Inv} 15$ require respectively the lemmas S1Inv13,..., S4Inv13. Likewise, item 11 has been directly put into Inv14. The most delicate to be proven was S2Inv13.

\subsection{Lemma 6.1}

The formalisation of Lemma 6.1 directly follows the text given in Section 6. So, it has been split into three lemmas Item1lemma6_1, Item2lemma6_1, and Item3lemma6_1. We present here only the first one.

Item1lemma6_1 : LEMMA FORALL ( $\mathrm{k}$ : nat,t: intlist, $n$ : listlist,

$\operatorname{Inv}(k, t, n, p, w, s)$ AND $s(0)=1 \Rightarrow$ $\mathrm{p}$ : boundintlist, w: intlist,s: statelist) :

FORALL (i: upto $(n b)): F C 2(k, t, n, p, w, s)$ AND $s(i)=1 \Rightarrow n(i)=n u l l$ 
where $\mathrm{FC} 2$ defined below corresponds to the optimised focus condition $F C$ introduced in Section 6 .

$\mathrm{FC2}$ (k: nat,t: intlist, $\mathrm{n}$ : listlist,p: boundintlist,w: intlist,s: statelist): bool $=$ FORALL $(i, j: \operatorname{upto}(n b)):$

$(s(i)=2$ or not (member $(i, n(j)))$ or $s(j) /=1$ or $i=j)$

and

$(n(j) /=n u l l$ or $w(j)>0$ or $s(j) /=1$ or $s(p(j)) /=1$ or $j=0)$.

- Consider, for example, the following part of the proof of Item2lemma6.1, corresponding to Lemma 6.1.2. Under the hypotheses $\operatorname{Inv}\left(\boldsymbol{n}_{0}, \boldsymbol{t}_{0}, n, \boldsymbol{t}, \boldsymbol{n}, \boldsymbol{p}, \boldsymbol{w}, \boldsymbol{s}\right), \boldsymbol{s}[0]=1, F C(n, \boldsymbol{t}, \boldsymbol{n}, \boldsymbol{p}, \boldsymbol{w}, \boldsymbol{s})$, $\boldsymbol{w}[0]>0$, we can construct for any integer $m$, a sequence of $m+1$ processes $0=i_{0}, i_{1}, \ldots, i_{m}$ such that for all $0 \leq l \leq m, s\left[i_{l}\right]=1, w\left[i_{l}\right]>0, p\left[i_{l+1}\right]=i_{l}$, and if $l \neq 0, i_{l} \neq 0$.

The construction of the sequence is formalised by the lemma Constructsequel. It turned out to be convenient to use the relation $\boldsymbol{p}^{h}\left[i_{0}\right]=i_{h}$, i.e. $i_{h}$ is the $h^{\text {th }}$ successor of $i_{0}$. Using the function iterate, $\boldsymbol{p}^{h}\left[i_{0}\right]=i_{h}$ is modeled by iterate $(\mathrm{p}, \mathrm{h})(\mathrm{i})$.

ConstructSequel : LEMMA FORALL ( $\mathrm{k}$ : nat, $\mathrm{t}$ : intlist, $\mathrm{n}$ : listlist,

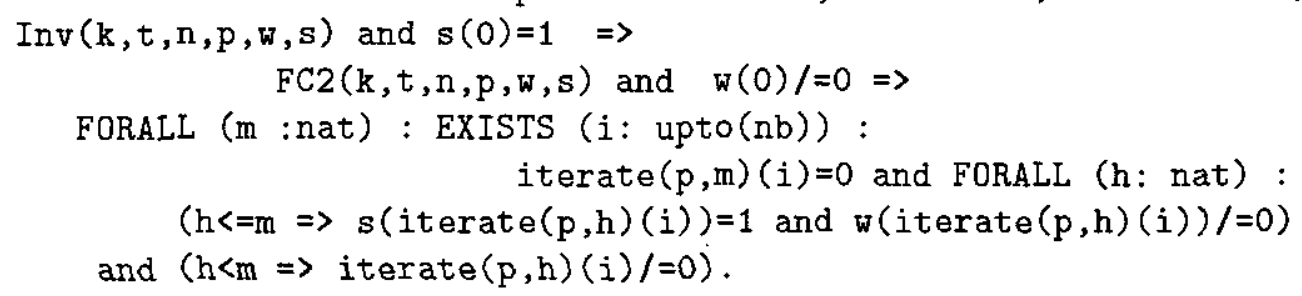

Next, consider a step of the proof of Lemma 6.1.3 under the following assumptions:

$\operatorname{Inv}\left(\boldsymbol{n}_{0}, \boldsymbol{t}_{0}, n, t, \boldsymbol{n}, \boldsymbol{p}, \boldsymbol{w}, \boldsymbol{s}\right), \boldsymbol{s}[0]=1, \boldsymbol{w}[0]=0$, the existence of a process $i \neq 0$ with $\boldsymbol{s}[i]=0$, and the existence of a sequence $i=i_{0}, \ldots, i_{m}=0$ such that for all $0 \leq l<m, i_{l+1} \in n_{0}\left[i_{l}\right]$. We have to prove that $s\left[i_{h}\right]=0$ for all $h, 0 \leq h \leq m$. This is obtained via the following lemma:

BuiltNewSequel : LEMMA FORALL ( $\mathrm{k}$ : nat, $\mathrm{t}$ intlist, $\mathrm{n}$ : listlist, $\mathrm{p}$ : boundintlist,w: intlist, $\mathrm{s}$ : statelist) :

$\operatorname{Inv}(k, t, n, p, w, s)$ AND $s(0)=1$ AND $w(0)=0 \Rightarrow$

FORALL (i,m: upto(nb), fm: $[u p t o(m) \rightarrow \operatorname{upto}(n b)])$ :

$(i /=0$ AND $s(i)=0$ AND $f m(0)=i$ AND $f m(m)=0$ AND

FORALL ( $I$ : upto $(m)): I<m \Rightarrow$ member $(f m(l+1)$, no $(f m(l)))$ )

$\Rightarrow$ FORALL $(h:$ upto $(m)): s(f m(h))=0$

where $\mathbf{f m}(h)$ stands for $i_{h}$. The proof of BuiltNewsequel requires in particular the fact that any process of the sequence mentioned above can't be in state 1 . This is provided by the first step of the proof of Lemma 6.1.3, and corresponds to the following lemma:

StepforItem3 : LEMMA FORALL ( $\mathrm{k}$ : nat,t: intlist, $\mathrm{n}$ : listlist,p: boundintlist, $\mathrm{w}$ : intlist,s: statelist) :

$\operatorname{Inv}(k, t, n, p, w, s)$ AND $s(0)=1$ AND $w(0)=0 \Rightarrow$

FORALL (i: upto $(n b)): i /=0 \Rightarrow s(i) /=1$ 


\section{COMPUTER-CHECKING THE VERIFICATION}

On the whole, the formalised proof of Item2lemma6_1 required 2 auxiliary definitions and 14 lemmas. Some of them were also used at other places, in particular for Item31emma6_1, which required overall 6 lemmas.

\subsection{State mapping, focus points and matching criteria}

As explained in Section 6, the state mapping $\mathrm{h}:[D \mathrm{DX} \rightarrow \mathrm{DY}]$ occurring in the theorem GET is provided by the following function

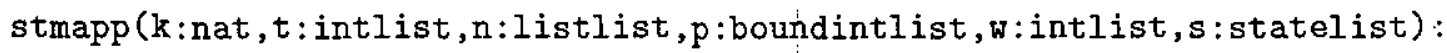

$$
\text { bool }=s(0)=1 \text {. }
$$

The application of the theorem GET in the proof of MAINTHM leads to following proof six obligations Convx (L_Impl), Crit2(L_Impl,d, stmapp), Crit3(L_Impl,L_Spec, d, stmapp),..., Crit6 (L_Impl, L_Spec, d, stmapp) to be proven using the topological hypotheses and the invariant Inv (d). Corresponding to these proof obligations, we define six predicates criter 1 , ..., criter6 and introduce six lemmas Invcriter $1, \ldots$, Invcriter 6 . The predicates correspond respectively to the formalisation of the items given in the proof of Lemma 6.2. For example the fifth criterion is:

criter5( $\mathrm{k}$ : nat, $\mathrm{t}:$ intlist, $\mathrm{n}:$ listlist, $\mathrm{p}$ : boundintlist, $\mathrm{w}$ : intlist, $\mathrm{s}: \mathrm{statelist):}$ bool $=n(0)=n u l l$ and $w(0)=0$ and $s(0)=1 \Rightarrow t(0)=$ totsum(to)

The lemmas Invcriter1, ..., Invcriter6 assert that each criterion holds under Inv. Note that criter 5 is the only criterion which requires the topological hypotheses:

Invcriter5 : LEMMA FORALL ( $\mathrm{k}$ : nat, $\mathrm{t}$ : intlist, $\mathrm{n}$ : listlist, $\mathrm{p}$ : boundintlist, w: intlist, $\mathrm{s}$ : statelist) :

pretopology and $\operatorname{Inv}(k, t, n, p, w, s) \Rightarrow \operatorname{criter} 5(k, t, n, p, w, s)$.

During the proof of MAINTHM, Crit5(L_Impl,L_Spec,d,stmapp) was smoothly reduced to proving criter5(proj_1(d), ..., proj_6(d)) for which we could use the lemma Invcriter5 mentioned above. The others were proven in the same way. As a detail, we mention that, whereas Crit4 (L_Impl, L_Spec, d, stmapp) mentions the focus condition FC(L_Impl,d), we use for its proof Lemma 6.1 which used the optimised focus condition FC2(d). To bridge this gap, we provided an auxiliary focus point formula FC1, defined below, together with a lemma FCequivFC1 which shows that FC is equivalent to FC1. Next we proved, assuming the invariant Inv, lemma FC1equivFC2 which establishes the equivalence between FC1 and FC2 and so between FC and FC2.

FC1 (k: nat, $t$ : intlist, $n:$ listlist,p: boundintlist, $w$ : intlist, $s$ : statelist):

bool $=($ FORALL $(i, j: \operatorname{upto}(n b)):$ $\operatorname{not}(s(i)=0$ and member $(i, n(j))$ and $s(j)=1$ and $i /=j))$

and (FORALL ( $i, j:$ upto $(n b))$ : $\operatorname{not}(s(i)=1$ and member $(i, n(j))$ and $s(j)=1$ and $i /=j))$

and (FORALL $(i, j:$ upto $(n b))$ :

$\operatorname{not}(n(j)=\operatorname{null}$ and $w(j)=0$ and $s(j)=1$ and $s(p(j))=1$ and $j /=0$ and $j /=p(j))$ ). 
Below we give the lemma FC1equivfC2. Actually, its proof require the following lemma noclon which is easily proven. It asserts that if $i$ can reach to another process $j$ via parent links, then $i$ can't be its own parent.

FC1equivFC2 : LEMMA FORALL ( $k$ : nat, $t$ : intlist, $n$ : listlist,p: boundintlist, w: intlist, $s:$ statelist) :

$\operatorname{Inv}(k, t, n, p, w, s) \Rightarrow(F C 1(k, t, n, p, w, s) \Leftrightarrow F C 2(k, t, n, p, w, s))$

noclon : LEMMA FORALL ( $i, j:$ upto(nb),p: boundintlist,m: nat) : $\operatorname{preach}(i, j, p, m)$ and $i /=j \Rightarrow p(i) /=i$.

where preach $(i, j, p, m)$ represents $\operatorname{Preach}(i, j, p, m)$ (Definition 5.1).

\subsection{Final steps of the proof}

We finish the formal proof using the main steps of the previous subsections and we show how the last arguments of the proof of Theorem 6.3 given in Section 6 have been translated in PVS.

The first required step before applying the theorem GET is to make sure that L_Impl and $\mathrm{L} \_$Spec are linear process equations. This is the same as establishing that they are respectively of the types LPE and ALPE. In other words, the LPEs properties arise as types correctness conditions.

As Theorem 6.3 is a consequence of the General Equality Theorem A.3, we find on the top of the proof commands tree of MAINTHM the two following commands (LEMMA "GET") introducing the theorem GET in the proof of MAINTHM, and (INST -1 "L_Impl" "L_Spec" "stmapp" "Inv") instantiating the quantifiers of GET. Next, the hypothesis goodtopology in MAINTHM has been put as an antecedent in the sequent formalizing the main theorem. Finally the assumptions of GET have been split off, providing the following formula.

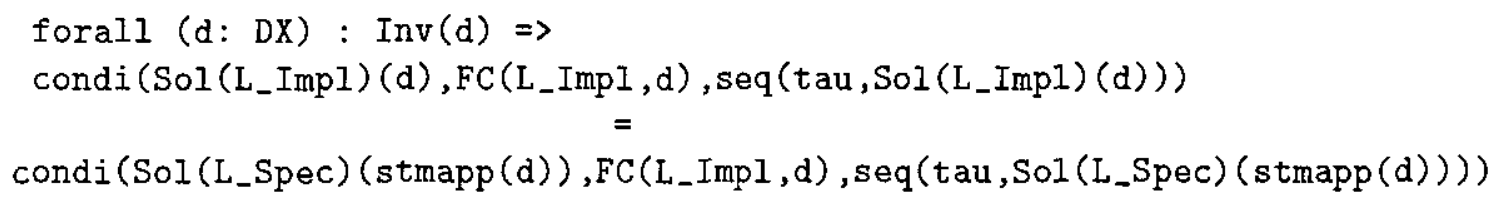

as an antecedent from which we derive the main theorem below, and returning the formulas Invlpox (L_Impl, Inv) and

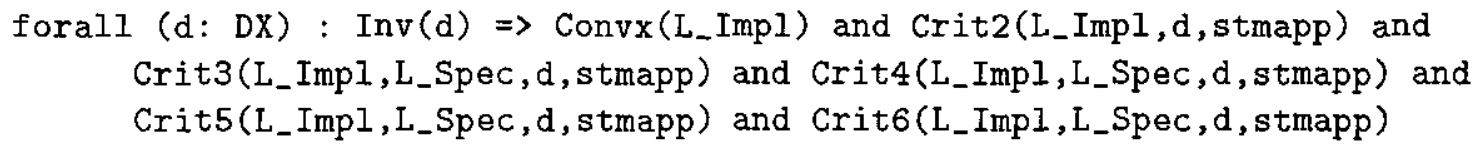

of GET as two new proof obligations. The first one of these has been proved as described in subsection 7.3. Next, we have skolemised the second formula, moved the hypothesis Inv as antecedent and split the resulting formula into six sequents. Each of them could be proven as described in subsection 7.5 .

This leaves the main sequent to be proved. The quantified variable $d$ in the antecedent coming from GET mentioned above was instantiated with ( $\mathrm{nb}$, to, no, po, wo, so). The lemma 


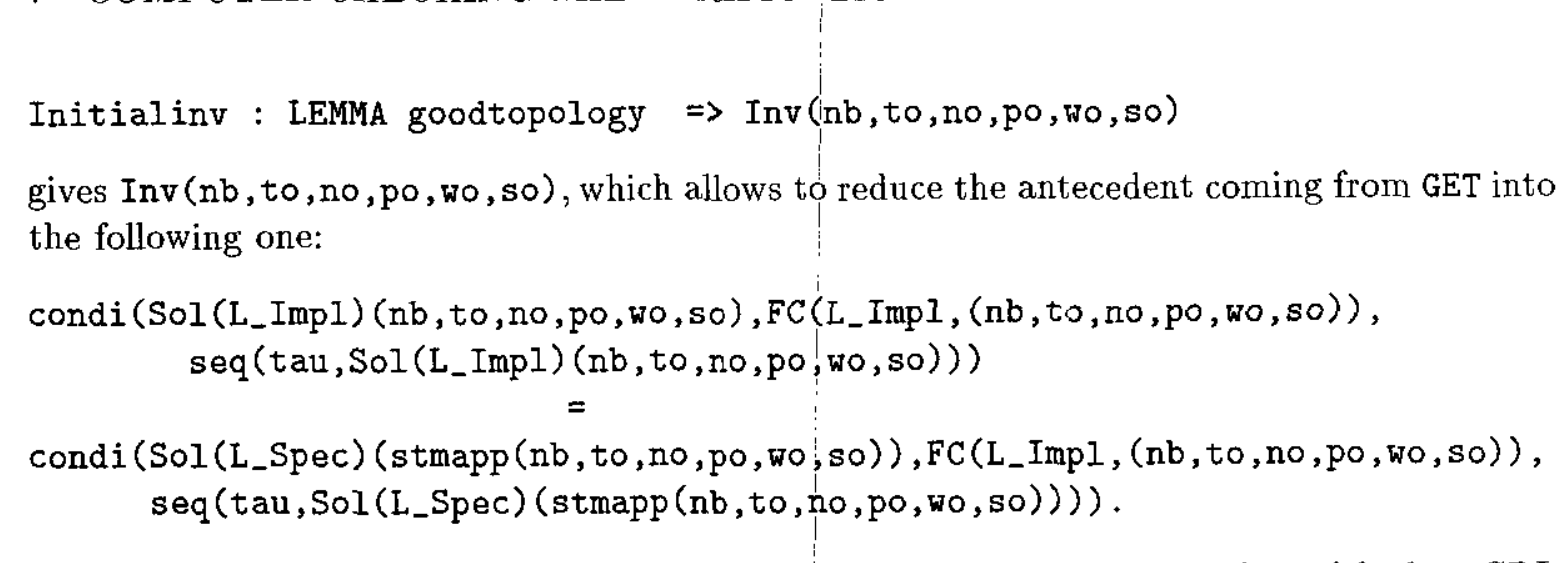

Next, we used the stmapp function, the so value, and Sol (L_Spec) together with the $\mu \mathrm{CRL}$ axioms to obtain

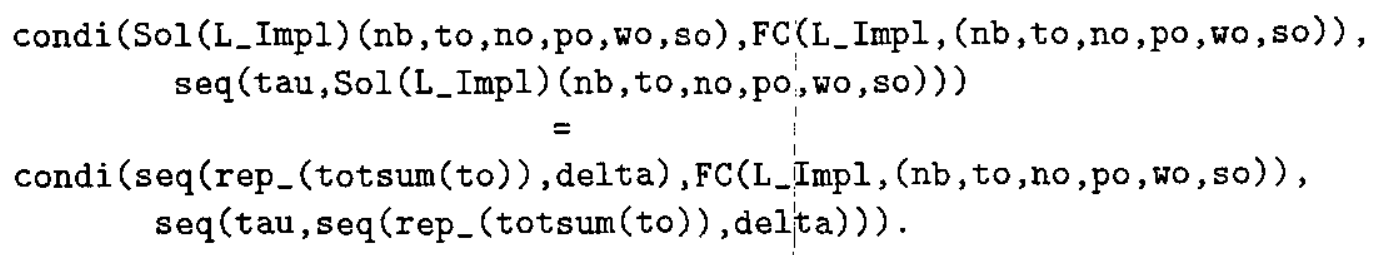

We then proceeded with a case distinction between FC(L_Impl, (nb, to, no, po, wo, so)) being true and false, respectively. Finally, we introduced the following lemma:

ReduceFC : LEMMA pretopology $\Rightarrow$

(FC2(nb, to, no,po,wo, so) iff FORALL (i:upto(nb)) : not(member(i,no(0))))

This allowed us to establish MAINTHM and therefore to finish the proof: Q.E.D.

\subsection{Discussion}

The distributed summation algorithm has been computer checked with on the whole 134 lemmas. Apart from the Type Correctness Conditions (T.C.C.s) concerning the LPEs L_ImpI and L_Spec, this does not take into account the OBLIGATIONs lemmas, since these were generated automatically by PVS for the T.C.C.s so did not have to be devised, and were always immediately proven. The complete proof development (definitions, lemmas including OBLIGATION lemmas also, and proof scripts) comprises about $270 \mathrm{~Kb}$.

The expressive power of the PVS system allowed us to translate the definitions and lemmas in an accurate way. However it turned out that it was difficult to translate the general notion of a linear process equation (LPE). The reason is that its definition is complex and polymorphic, involving data types as parameters. In particular a family of a priori different types indexed by a finite set of actions appears in the LPEs. This phenomenon also occurs in the matching criteria of the General Equality Theorem. Therefore we have explicitly introduced a number bounding the size of the set of actions which allowed us to use record types for LPEs instead of functional definitions.

As mentioned at the beginning of this section, the theorem GET has been provided as an axiom in PVS and, being part of the meta-theory of $\mu \mathrm{CRL}$, was not mechanically checked itself. We point out that the theorem MAINTHM has been proven without any axioms other 
than an axiom stating that $\overline{r e p}$ action and $\tau$ action are distinct, those of $\mu$ CRL and GET within the logical framework of PVS.

As has been illustrated with the distributed summation algorithm, we think that the syntactical and axiomatic description in $\mu \mathrm{CRL}$ of distributed systems is suitable for verification and enables the proofs to be checked by higher order proof checkers or theorem provers such as PVS and COQ leading to an extremely high level of confidence in the correctness of the proofs.

\section{Comparison with other verifications.}

Our appraisal of the applicability of formal techniques for reasoning about distributed algorithms differs strongly from Chou's. We feel that proof techniques from the area of formal methods are sufficiently mature to prove the correctness of protocols of at least the complexity of a distributed summation algorithm. We are convinced that the reader - after having read, digested and understood the correctness proof - will agree that it is straightforward and not at all more complex than necessary.

There are as far as we know three other formal proofs of the distributed summation algorithm. In [18] Vaandrager proves the summation algorithm correct in the setting of I/O automata. His description of the algorithm, which is best compared to the linearisation of the algorithm in Table 1, differs from ours in two aspects. First, in his set-up processes communicate asynchronously by means of queues, whereas we let processes communicate using synchronous interaction. The second difference is that in [18] when a process reads a $\overline{s t}$ message from its input queue, $\overline{s t}$ messages are put simultaneously in all outgoing queues, whereas in our setting sending these messages happens in an interleaved way.

The structure of Vaandrager's proof is the following. First, some invariants are proven. Using these, a relation is defined between implementation and specification that is proven to be a refinement. From this it may be concluded that the trace set of the implementation is included in the trace set of the specification. As trace inclusion does not imply deadlockfreeness, this fact is proven separately.

There are two major differences between both proofs. In [18] history and prophecy variables are employed which are not present in our paper. It is remarked in [18] that it should be possible to give the proof without such auxiliary variables, but that they have been included to illustrate their use. Secondly, although the refinement that is presented is very much like our state mapping $h$, we establish branching bisimulation between specification and the algorithm, whereas using the refinement, only a weaker fact, namely trace inclusion is shown. Therefore, we do not have to show deadlock freeness separately, as branching bisimulation preserves deadlock freeness.

It is also important to note the similarities between both proofs. The overall structure of the proofs is the same, as are the essential arguments. Actually, it would not be very hard to upgrade the proofs of trace inclusion and deadlock freeness in [18] to imply a result such as ours.

The description of the algorithm by Chou [2] closely resembles the description of [18]. Chou's proof sets out with defining three modal properties together stating that the algorithm will deliver the total sum exactly once. First, it is argued that proving the modal properties 
directly on the description of the distributed summation algorithm is too complicated. Then a more abstract version of the algorithm is defined in terms of causes and events, the state space of which can be characterised by simple invariants. The abstract version is related to the original one by means of a simulation relation and a 'joint invariant'. It is shown that translated versions of the modal correctness properties hold for the abstract version. Using the simulation relation and the joint invariant it is shown that validity of the original correctness properties can be derived for the original algorithm. Chou's proof thus is similar to Vaandrager's proof except that correctness is stated by means of modal properties instead of by a specification automaton, and the abstract version is defined in terms of causes and events. To the best of our knowledge, these proofs have not been proof-checked.

We remark that our proof method is purely! syntactical and axiomatic, while the proofs in $[2,18]$ have a semantical nature. This is not very visible in this paper, as we have for readability omitted all syntactic definitions of data types and employ the General Equality Theorem from [8] whose proof is syntactical but which has a semantic flavour. We feel that our method shares the advantages of semantical reasoning, while its axiomatic nature allows a complete, computer-checked formalisation.

A third proof of essentially the same description of the protocol as the one of Chou and Vaandrager is given by Hesselink [9]. He describes the protocol using LISP functions that are triggered by data in input queues and atomically put data in all output queues of a process. In order to model non-deterministic behaviour, Hesselink introduces an oracle. He then proves that the protocol terminates and that if terminated the total sum is collected in the root. These observations exactly matchi with proof steps one and five of Lemma 6.2. Hesselink uses the Boyer-Moore theorem prover to verify the correctness of his proofs.

\section{A Short description of $\mu \mathrm{CRL}$}

The language $\mu \mathrm{CRL}$ is a formalism (with proof theory) for process algebra comprising data $[7,6]$. In this section we give a brief overview of the $\mu$ CRL syntax for processes and restate the General Equality Theorem of [8], which is the basis of the correctness proof in this paper. In order to do the latter we have to define the:format for linear process equations.

\section{A.1 Overview of syntax}

Starting from a set Act of actions that can be parameterised with data, processes are defined by means of guarded recursive equations and the following operators.

First, there is a constant $\delta(\delta \notin \mathrm{Act})$ that cannot perform any action and is called deadlock or inaction.

Next, there are the sequential composition operator $\cdot$ and the alternative composition operator + . The process $x \cdot y$ first behaves as $x$ and if $x$ successfully terminates continues to behave as $y$. The process $x+y$ can either do an action of $x$ and continue to behave as $x$ or do an action of $y$ and continue to behave as $y$;

Interleaving parallelism is modeled by the operator $\|$. The process $x \| y$ is the result of interleaving actions of $x$ and $y$, except that actions from $x$ and $y$ may also synchronise to a communication action, when this is explicitly allowed by a communication function. This is a partial, commutative and associative function $\gamma:$ Act $\times$ Act $\rightarrow$ Act that describes how actions 
can communicate; parameterised actions $a(d)$ and $b\left(d^{\prime}\right)$ communicate to $\gamma(a, b)(d)$, provided $d=d^{\prime}$. A specification of a process typically contains a specification of a communication function.

In order to axiomatise the parallel operator there are two auxiliary parallel operators. First, the left merge $\mathbb{L}$, which behaves as the parallel operator, except that the first step must come from the process at the left. Secondly, the communication merge / which also behaves as the parallel operator, except that the first step is a communication between both arguments, as specified by the communication function $\gamma$. We often write $a \mid b=c$ for $\gamma(a, b)=c$.

To enforce that actions in processes $x$ and $y$ synchronise, we can prevent actions from happening on their own, using the encapsulation operator $\partial_{H}$. The process $\partial_{H}(x)$ can perform all actions of $x$ except that actions in the set $H$ are blocked. So, assuming $\gamma(a, b)=c$, in $\partial_{\{a, b\}}(x \| y)$ the actions $a$ and $b$ are forced to synchronise to $c$.

We assume the existence of a special action $\tau$ ( $\tau \notin$ Act) that is internal and cannot be directly observed. The hiding operator $\tau_{I}$ renames the actions in the set $I$ to $\tau$. By hiding all internal communications of a process only the external actions remain.

The following two operators combine data with processes. The sum operator $\Sigma_{d: D} p(d)$ describes the process that can execute the process $p(d)$ for some value $d$ selected from the sort $D$. The conditional operator $\_\triangleleft \_\_$describes the then-if-else. The process $x \triangleleft b \triangleright y$ (where $b$ is a boolean) has the behaviour of $x$ if $b$ is true and the behaviour of $y$ if $b$ is false. When the right hand side trivialises, i.e. $y$ equals $\delta$, we write $[b] \Rightarrow x$.

We apply the convention that $\cdot$ binds stronger than $\Sigma$, followed by $-\triangleleft_{-} \Delta_{-}$, the parallel operators, and + binds weakest. Moreover, - is usually suppressed.

We work in the setting of branching bisimulation [17], which is a refinement of weak bisimulation [11].

Axioms for the operators can be found, e.g., in [7].

\section{A.2 Linear process equations}

The process equations for process $P$ in Definition 3.1 and for $L$-Impl in Table 1 are (essentially) written in the format of linear process equations (LPEs). A linear process equation is of the form $X(d: D)=R H S$, where $d$ is a parameter of type $D$ and $R H S$ consists of an alternative composition of a number of summands of the form

$$
\sum_{e: E}[b(d, e)] \Rightarrow a(f(d, e)) X(g(d, e))
$$

Such a summand means that if for some $e$ of type $E$ the guard $b(d, e)$ is satisfied, the action $a$ can be performed with parameter $f(d, e)$, followed by a recursive call of $X$ with new value $g(d, e)$. Now the main feature of LPEs is that for each action there is a most one summand in the alternative composition ${ }^{1}$. This makes it possible to describe LPEs by means of a finite set $A c t$ of actions as indices, giving for each action $a$ the set $E_{a}$ over which summation takes place, the guard $b_{a}$ that enables the action, the function $f_{a}$ that determines the data parameter of the action and the function $g_{a}$ that determines the value of the recursive call.

In the next definition the symbol $\Sigma$, used for summation over data types, is also used to describe an alternative composition over a finite set of actions. If $A c t=\left\{a_{1}, \ldots, a_{n}\right\}$, then

\footnotetext{
${ }^{1}$ The LPEs described here, are called deterministic in [8].
} 
$\Sigma_{a \in A c t} p_{a}$ denotes $p_{a_{1}}+p_{a_{2}}+\cdots+p_{a_{n}}$. Note that for summation over actions the symbol $\epsilon$ is used (instead of the symbol :).

Definition A.1. Let $A c t \subseteq$ Act $\cup\{\tau\}$ be a finite set of actions, and let $D$ be a data type. A linear process equation ( $L P E$ ) over Act and $D$ is an equation of the form

$$
X(d: D)=\sum_{a \in A c t} \sum_{e: E_{a}}\left[b_{a}(d, e)\right] \Rightarrow a\left(f_{a}(d, e)\right) X\left(g_{a}(d, e)\right)
$$

for some data types $E_{a}, D_{a}$, and functions $f_{a}: D \rightarrow E_{a} \rightarrow D_{a}, g_{a}: D \rightarrow E_{a} \rightarrow D$, $b_{a}: D \rightarrow E_{a} \rightarrow$ Bool. (We assume that $\tau$ has no parameter.)

The process equations for process $P$ in Definition 3.1 and for $L$ - $I m p l$ in Table 1 do not directly fit in the LPE format; consult [8] to verify that the deviations are harmless.

Definition A.2. An LPE $X$ written as in Definition A.1 is called convergent if it does not admit infinite $\tau$-paths, i.e., there is a well-founded ordering $<$ on $D$ such that for all $e: E_{\tau}$ and $d: D$ we have that $b_{\tau}(d, e)$ implies $g_{\tau}(d, e)<d$.

An invariant of an LPE $X$ written as in Definition A.1 is a function $I: D \rightarrow$ Bool such that for all $a \in A c t, e: E_{a}$, and $d: D$ we have $b_{a}(d, e) \wedge I(d) \rightarrow I\left(g_{a}(d, e)\right)$.

For each LPE $X$, we assume an axiom which postulates that $X$ has a solution, and an axiom that postulates that every convergent LPE has at most one solution. In this way, convergent LPEs define processes. The two principles reflect that we only consider process algebras where every LPE has at least one solution and converging LPEs have precisely one solution.

\section{A.3 General Equality Theorem}

Theorem A.3 (General Equality Theorem from [8]). Let $X$ and $Y$ be LPEs given as follows:

$$
\begin{aligned}
& X\left(d: D_{X}\right)=\sum_{a \in A c t} \sum_{e: E_{a}}\left[b_{a}(d, e)\right] \Rightarrow a\left(f_{a}(d, e)\right) X\left(g_{a}(d, e)\right) \\
& Y\left(d: D_{Y}\right)=\sum_{a \in A c t \backslash\{\tau\}} \sum_{e: E_{a}}\left[b_{a}^{\prime}(d, e)\right] \Rightarrow a\left(f_{a}^{\prime}(d, e)\right) Y\left(g_{a}^{\prime}(d, e)\right)
\end{aligned}
$$

Let $F C_{X}$ be a formula over $d: D_{X}$ describing exactly the states of $X$ from which no $\tau$-action is enabled (i.e. equivalent to $\neg \exists x: E_{\tau} b_{\tau}(d, x)$ ). . Assume that $r$ and $q$ are solutions of $X$ and $Y$, respectively. Suppose $I$ is an invariant of $X \mid$ and, for all $d: D_{X}, I(d)$ implies the following set of matching criteria.

$X$ is convergent

$$
\begin{aligned}
& \forall e: E_{\tau}\left(b_{\tau}(d, e) \rightarrow h(d)=h\left(g_{\tau}(d, e)\right)\right) \\
& \forall a \in A c t \backslash\{\tau\} \forall e: E_{a}\left(b_{a}(d, e) \rightarrow b_{a}^{\prime}(h(d) ; e)\right) \\
& \forall a \in A c t \backslash\{\tau\} \forall e: E_{a}\left(F C_{X}(d) \wedge b_{a}^{\prime}(h(d), e) \rightarrow b_{a}(d, e)\right)
\end{aligned}
$$




$$
\begin{aligned}
& \forall a \in A c t \backslash\{\tau\} \forall e: E_{a}\left(b_{a}(d, e) \rightarrow f_{a}(d, e)=f_{a}^{\prime}(h(d), e)\right) \\
& \forall a \in A c t \backslash\{\tau\} \forall e: E_{a}\left(b_{a}(d, e) \rightarrow h\left(g_{a}(d, e)\right)=g_{a}^{\prime}(h(d), e)\right)
\end{aligned}
$$

Then

$\forall d: D_{X} I(d) \rightarrow r(d) \triangleleft F C_{X}(d) \triangleright \tau r(d)=q(h(d)) \triangleleft F C_{X}(d) \triangleright \tau q(h(d))$.

\section{References}

[1] J.C.M. Baeten and W.P. Weijland. Process Algebra, volume 18 of Cambridge Tracts in Theoretical Computer Science. Cambridge University Press, 1990.

[2] C.-T. Chou. Practical use of the notions of events and causality in reasoning about distributed algorithms. CS Report \#940035, UCLA, October 1994.

[3] C. Cornes et al. The Coq Proof Assistant Reference Manual, Version 5.10. Technical Report, INRIA, 1996.

[4] E.W. Dijkstra and C.S. Scholtens. Termination detection for diffusing computations. Inf. Processing Letters, 11(1):1-4, 1980.

[5] J.F. Groote. A note on $n$ similar processes. Technical report CS-R9626, Department of Software Technology, CWI, Amsterdam, 1996, June 1996.

[6] J.F. Groote and A. Ponse. The syntax and semantics of $\mu$ CRL. In A. Ponse, C. Verhoef and S.F.M. van Vlijmen, eds, Algebra of Communicating Processes, Workshops in Computing, pp. 26-62, 1994.

[7] J.F. Groote and A. Ponse. Proof theory for $\mu$ CRL: a language for processes with data. In Andrews et al. Proceedings of the International Workshop on Semantics of Specification Languages. Workshops in Computing, pages 231-250. Springer Verlag, 1994.

[8] J.F. Groote and J. Springintveld. Focus points and convergent process operators. A proof strategy for protocol verification. Technical Report 142, Logic Group Preprint Series, Utrecht University, 1995. This report also appeared as Technical Report CSR9566, Centrum voor Wiskunde en Informatica, 1995

[9] W.H. Hesselink. A mechanical proof of Segall's PIF algorithm. Formal Aspects of Computing, 9(2), pages 208-226, 1997.

[10] H. Korver and A. Sellink. On automating process algebra proofs. In V. Atalay et. al., editors, Proceedings of the 11-th International Symposium on Computer and Information Sciences, ISCIS XI, Antalya, Turkey, volume II, pages 815-826, November 1996 
[11] R. Milner. Communication and Concurrency. Prentice Hall, London, 1989.

[12] S. Owre, J.M. Rushby, N. Shankar and F. von Henke. Formal verification for faulttolerant archictectures: Prolegomena to the design of PVS. IEEE Transactions on Software Engineering, 21(2): 107-125, 1995.

[13] S. Owre, N. Shankar and J.M. Rushby. The PVS Specification Language. Computer Science Laboratory, SRI International, Menlo Park, CA, February 1993.

[14] S. Owre, N. Shankar and J.M. Rushby; User Guide for the PVS Specification and Verification System. Computer Science Laboratory, SRI International, Menlo Park, CA, February 1993.

[15] S. Segall. Distributed network protocols. IEEE Transactions on Information Theory, IT-29(2):23-35, 1983.

[16] N. Shankar, S. Owre and J.M. Rushby. The PVS Proof Checker: A Reference Manual. Computer Science Laboratory, SRI International, Menlo Park, CA, February 1993.

[17] R.J. van Glabbeek and W.P. Weijland. Branching time and abstraction in bisimulation semantics (extended abstract). In G.X. Ritter, editor, Information Processing 89, pages $613-618,1989$.

[18] F.W. Vaandrager. Verification of a distributed summation algorithm. In I. Lee and S.A. Smolka, editors, Proceedings CONCUR95, pages 190-203, LNCS 962, SpringerVerlag, 1995. 


\section{Computing Science Reports}

\section{In this series appeared:}

\begin{tabular}{|c|c|}
\hline $96 / 01$ & M. Voorhoeve and T. Basten \\
\hline $96 / 02$ & P. de Bra and A. Aerts \\
\hline $96 / 03$ & W.M.P. van der Aalst \\
\hline $96 / 04$ & S. Mauw \\
\hline $96 / 05$ & T. Basten and W.M.P. v.d. Aalst \\
\hline $96 / 06$ & W.M.P. van der Aalst and T. Basten \\
\hline $96 / 07$ & M. Voorhoeve \\
\hline $96 / 08$ & $\begin{array}{l}\text { A.T.M. Aerts, P.M.E. De Bra, } \\
\text { J.T. de Munk }\end{array}$ \\
\hline $96 / 09$ & F. Dignum, H. Weigand, E. Verharen \\
\hline $96 / 10$ & R. Bloo, H. Geuvers \\
\hline $96 / 11$ & T. Laan \\
\hline $96 / 12$ & F. Kamareddine and T. Laan \\
\hline $96 / 13$ & T. Borghuis \\
\hline $96 / 14$ & S.H.J. Bos and M.A. Reniers \\
\hline $96 / 15$ & M.A. Reniers and J.J. Vereijken \\
\hline $96 / 17$ & E. Boiten and P. Hoogendijk \\
\hline $96 / 18$ & P.D.V. van der Stok \\
\hline $96 / 19$ & M.A. Reniers \\
\hline $96 / 20$ & L. Feijs \\
\hline $96 / 21$ & L. Bijlsma and R. Nederpelt \\
\hline $96 / 22$ & M.C.A. van de Graaf and G.J. Houben \\
\hline $96 / 23$ & W.M.P. van der Aalst \\
\hline $96 / 24$ & M. Voorhoeve and $W$. van der Aalst \\
\hline $96 / 25$ & M. Vaccari and R.C. Backhouse \\
\hline $97 / 01$ & B. Knaack and R. Gerth \\
\hline $97 / 02$ & J. Hooman and $O . v$. Roosmalen \\
\hline $97 / 03$ & J. Blanco and A. v. Deursen \\
\hline $97 / 04$ & J.C.M. Baeten and J.A. Bergstra \\
\hline $97 / 05$ & J.C.M. Baeten and J.J. Vereijken \\
\hline $97 / 06$ & M. Franssen \\
\hline $97 / 07$ & J.C.M. Baeten and J.A. Bergstra \\
\hline
\end{tabular}

\section{Department of Mathematics and Computing Science Eindhoven University of Technology}

Process Algebra with Autonomous Actions, p. 12.

Multi-User Publishing in the Web: DreSS, A Document Repository Service Station, p. 12

Parallel Computation of Reachable Dead States in a Free-choice Petri Net, $\beta .26$.

Example specifications in phi-SDL.

A Process-Algebraic Approach to Life-Cycle Inheritance Inheritance $=$ Encapsulation + Abstraction, p. 15.

Life-Cycle Inheritance A Petri-Net-Based Approach, p. 18.

Structural Petri Net Equivalence, p. 16.

OODB Support for WWW Applications: Disclosing the internal structure of Hyperdocuments, p. 14.

A Formal Specification of Deadlines using Dynamic Deontic Logic, p. 18.

Explicit Substitution: on the Edge of Strong Normalisation, p. 13.

AUTOMATH and Pure Type Systems, p. 30.

A Correspondence between Nuprl and the Ramified Theory of Types, p. 12.

Priorean Tense Logics in Modal Pure Type Systems, p. 61

The $I^{2}$ C-bus in Discrete-Time Process Algebra, p. 25.

Completeness in Discrete-Time Process Algebra, p. 139.

Nested collections and polytypism, p. 11.

Real-Time Distributed Concurrency Control Algorithms with mixed time constraints, p. 71.

Static Semantics of Message Sequence Charts, p. 71

Algebraic Specification and Simulation of Lazy Functional Programs in a concurrent Environment, p. 27.

Predicate calculus: concepts and misconceptions, p. 26.

Designing Effective Workflow Management Processes, p. 22.

Structural Characterizations of sound workflow nets, p. 22.

Conservative Adaption of Workflow, p.22

Deriving a systolic regular language recognizer, p. 28

A Discretisation Method for Asynchronous Timed Systems.

A Programming-Language Extension for Distributed Real-Time Systems, p. 50.

Basic Conditional Process Algebra, p. 20.

Discrete Time Process Algebra: Absolute Time, Relative Time and Parametric Time, p. 26.

Discrete-Time Process Algebra with Empty Process, p. 51.

Tools for the Construction of Correct Programs: an Overview, p. 33.

Bounded Stacks, Bags and Queues, p. 15. 
When do datatypes commute? p. 35

97/09 Proceedings of the Second International Workshop on Communication Modeling,

Communication Modeling- The Language/Action Perspective, p. 147 Veldhoven, The Netherlands, 9-10 June, 1997.

P.C.N. v. Gorp, E.J. Luit, D.K. Hammer E.H.L. Aarts

Distributed real-time systems: a survey of applications and a general design model, p. 31 .

97/11 A. Engels, S. Mauw and M.A. Reniers

A Hierarchy of Communication Models for Message Sequence Charts, p. 30.

$97 / 12$ D. Hauschildt, E. Verbeek and

W. van der Aalst

WOFLAN: A Petri-net-based Workflow Analyzer, p. 30.

97/13 W.M.P. van der Aalst

Exploring the Process Dimension of Workflow Management, p. 56. 
Technische Universiteit

Den Dolech 2

Postbus 513

$5600 \mathrm{MB}$ Eindhoven

Telefoon (040) 479111

Telex 51163

Telefax

$040-436685$

Datum

$\mathrm{MM} / \mathrm{TI}$ e-mail

wsinti@win.tue.nl

Doorkiesnummer

$040-474214$

Onderwerp

CSN-rapport

L.S.,

Bij deze verklaar $\mathrm{ik}$ dat het artikel

A computer checked algebraic verification of a distributed summation afgorithm

geschikt is om als CSN-rapport te verschijnen.

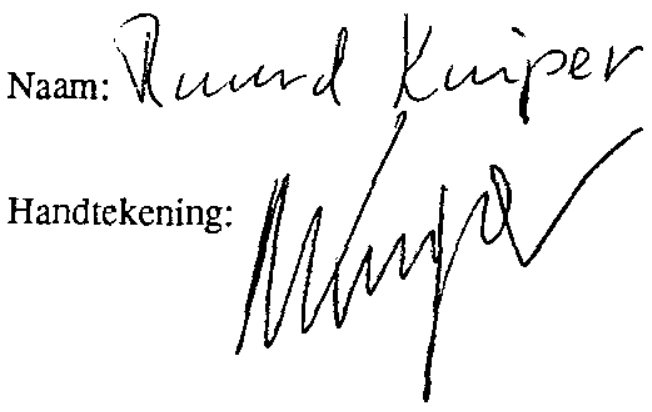


Technische Universiteit

Den Dolech 2

Postbus 513

$5600 \mathrm{MB}$ Eindhoven

Telefoon (040) 479111

Telex 51163

Uw kenmerk

Ons kenmerk

MM/TI
Telefax

$040-436685$

e-mail

wsinti@win.tue.nl

Datum

Doorkiesnummer

040 - 474214

Onderwerp

CSN-rapport

L.S.,

Bij deze verklaar ik dat het artikel

$$
\begin{aligned}
& \text { A computar checled algebmic ven fricatia } \\
& \text { ofa distibued }
\end{aligned}
$$

geschikt is om als CSN-rapport te verschijnen.

Naam:

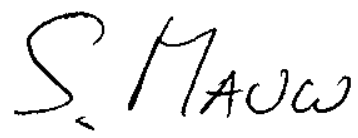

Handtekening:

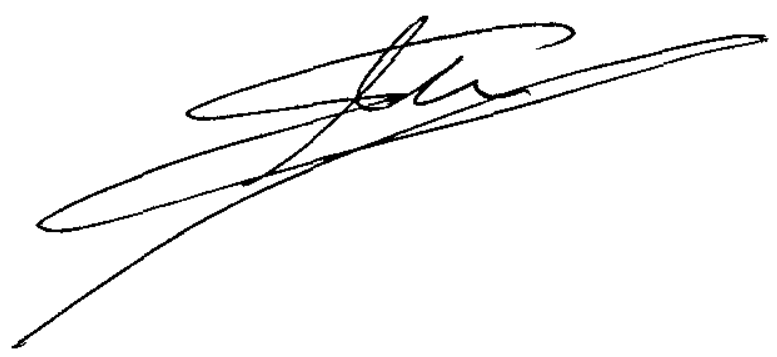

